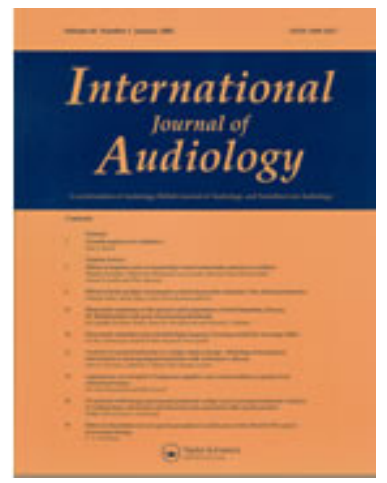

\title{
A process for prioritising systematic reviews in tinnitus
}

\begin{tabular}{|c|c|}
\hline Journal: & International Journal of Audiology \\
\hline Manuscript ID & TIJA-2019-10-0378.R1 \\
\hline Manuscript Type: & Technical Report \\
\hline $\begin{array}{r}\text { Date Submitted by the } \\
\text { Author: }\end{array}$ & $\mathrm{n} / \mathrm{a}$ \\
\hline Complete List of Authors: & $\begin{array}{l}\text { Sereda, Magdalena; University of Nottingham, NIHR Nottingham } \\
\text { Biomedical Research Centre, Hearing Sciences, Division of Clinical } \\
\text { Neuroscience, School of Medicine } \\
\text { McFerran, Don; Essex County Hospital, Otolaryngology; } \\
\text { Axon, Emma; University of Nottingham, Cochrane Skin, Centre of } \\
\text { Evidence Based Dermatology } \\
\text { Baguley, David; University of Nottingham, Hearing Sciences, NIHR } \\
\text { Nottingham Biomedical Research Centre, Hearing Sciences, Division of } \\
\text { Clinical Neuroscience, School of Medicine } \\
\text { Hall, Deborah; University of Nottingham, NIHR Nottingham Biomedical } \\
\text { Research Centre, Hearing Sciences, Division of Clinical Neuroscience, } \\
\text { School of Medicine; University of Nottingham - Malaysia Campus, Jalan } \\
\text { Broga, 43500 Semeniyh, Selangor Darul Ehsan } \\
\text { Potgieter, Iskra; University of Nottingham, NIHR Nottingham Biomedical } \\
\text { Research Centre, Hearing Sciences, Division of Clinical Neuroscience, } \\
\text { School of Medicine } \\
\text { Cima, Rilana; Adelante, Centre of expertise in Rehabilitation \& } \\
\text { Audiology; Maastricht University, Clinical Psychological Science } \\
\text { Cox, Samantha; University of Oxford, Cochrane ENT, Nuffield } \\
\text { Department of Surgery } \\
\text { Hoare, Derek; University of Nottingham, NIHR Nottingham Biomedical } \\
\text { Research Centre, Hearing Sciences, Division of Clinical Neuroscience, } \\
\text { School of Medicine }\end{array}$ \\
\hline Keywords: & Tinnitus, Instrumentation, Pharmacology, systematic review \\
\hline
\end{tabular}


56

57

58

59

60

E-mail:editor.ija@up.ac.za URL: http://mc.manuscriptcentral.com/tija 
Sereda et al. Prioritising topics for systematic review

\section{A process for prioritising systematic reviews in tinnitus}

\section{Technical report}

Magdalena Sereda ${ }^{1,2 *}$, Don McFerran ${ }^{3}$, Emma Axon ${ }^{4}$, David M Baguley ${ }^{1,2,5}$, Deborah A Hall ${ }^{1,2,5,6}$, Iskra Potgieter ${ }^{1,2}$, Rilana Cima ${ }^{7,8}$, Samantha Cox ${ }^{9}$, Derek J Hoare ${ }^{1,2}$

1 National Institute for Health Research (NIHR) Nottingham Biomedical Research Centre, Ropewalk House, 113 The Ropewalk, Nottingham, UK

2 Hearing Sciences, Division of Clinical Neuroscience, School of Medicine, University of Nottingham, Nottingham, UK

3 East Suffolk and North Essex NHS Foundation Trust, Colchester General Hospital, Turner Road, Colchester, UK

4 Cochrane Skin, Centre of Evidence Based Dermatology, University of Nottingham, Nottingham, UK

5 Nottingham University Hospitals NHS Trust, Queens Medical Centre, Derby Road, Nottingham, UK

6 University of Nottingham Malaysia, Jalan Broga, 43500 Semeniyh, Selangor Darul Ehsan, Malaysia

7 Maastricht University, Department of Clinical Psychological Science, Maastricht, Netherlands

8 Adelante, Centre for Expertise in Rehabilitation \& Audiology, Hoensbroek, Limburg, Netherlands

9 Cochrane ENT, Nuffield Department of Surgery, University of Oxford, Oxford, UK

*Corresponding author:

Magdalena Sereda

National Institute for Health Research (NIHR) Nottingham Biomedical Research Centre

Ropewalk House, 113 The Ropewalk

NG1 5DU, Nottingham, UK

Magdalena.Sereda@nottingham.ac.uk 
Sereda et al. Prioritising topics for systematic review

\begin{abstract}
Objective: To develop an innovative prioritisation process to identify topics for new or updated systematic reviews of tinnitus research.

Design: A two stage prioritisation process was devised. Firstly, a scoping review assessed the amount of randomised-controlled-trial-level evidence available. This enabled development of selection criteria for future reviews, aided the design of template protocol, and suggested the scale of work that would be required to conduct these reviews. Secondly, using the predefined primary and secondary criteria, interventions were prioritised for systematic review.
\end{abstract}

Study sample: Searches identified 1080 records. After removal of duplicates and out of scope works, 437 records remained for full data charting.

Results: The process was tested, using subjective tinnitus as the clinical condition and using Cochrane as the systematic review platform. The criteria produced by this process identified three high priority reviews: 1) Sound therapy using amplification devices and/or sound generators; 2) Betahistine, and 3) Cognitive Behaviour Therapy. Further secondary priorities were: 4) Gingko biloba, 5) Anxiolytics, 6) Hypnotics, 7) Antiepileptics, and 8) Neuromodulation.

Conclusions: A process was developed which successfully identified priority areas for Cochrane systematic reviews of interventions for subjective tinnitus. This technique could easily be transferred to other conditions and other types of systematic reviews. 
Keywords: Cochrane, systematic review, priority, management, treatment, tinnitus 
Sereda et al. Prioritising topics for systematic review

1 ABSTRACT

2 Objective: To develop an innovative prioritisation process to identify topics for new or

3 updated systematic reviews of tinnitus research.

4 Design: A two stage prioritisation process was devised. Firstly, a scoping review assessed the

5 amount of randomised-controlled-trial-level evidence available. This enabled development of

6 selection criteria for future reviews, aided the design of template protocol, and suggested the

7 scale of work that would be required to conduct these reviews. Secondly, using the pre-

8 defined primary and secondary criteria, interventions were prioritised for systematic review.

9 Study sample: Searches identified 1080 records. After removal of duplicates and out of

10 scope works, 437 records remained for full data charting.

11 Results: The process was tested, using subjective tinnitus as the clinical condition and using

12 Cochrane as the systematic review platform. The criteria produced by this process identified

13 three high priority reviews: 1) Sound therapy using amplification devices and/or sound

14 generators; 2) Betahistine, and 3) Cognitive Behaviour Therapy. Further secondary priorities

15 were: 4) Gingko biloba, 5) Anxiolytics, 6) Hypnotics, 7) Antiepileptics, and 8)

16 Neuromodulation.

17 Conclusions: A process was developed which successfully identified priority areas for

18 Cochrane systematic reviews of interventions for subjective tinnitus. This technique could

19 easily be transferred to other conditions and other types of systematic reviews.

22 Keywords: Cochrane, systematic review, priority, management, treatment, tinnitus 
Sereda et al. Prioritising topics for systematic review

\section{INTRODUCTION}

Systematic reviews and meta-analyses represent the highest level of evidence for the effectiveness of clinical interventions and hold a critical place in informing health policy and evidence-based practice (Greenwell et al.2016; Morata et al., 2017). One of the foremost organisations producing systematic reviews is Cochrane, which is a UK based charity (notfor-profit organisation) that supervises a global independent network of healthcare practitioners, researchers, patient advocates and others. It represents more than 11,000 members and over 68,000 supporters from over 130 countries

(https://www.cochrane.org/about-us). Cochrane authors conduct systematic reviews of health-care interventions and diagnostic tests which are published as Cochrane Reviews in the Cochrane Library. Previously, Cochrane authors self-selected topics for their reviews and submitted proposals to Cochrane for approval. This process has been updated and now, Cochrane groups are encouraged to work strategically to respond to the needs of funders and key stakeholders to produce reviews on topics of the highest priority to users. One approach to prioritising these reviews is to conduct a scoping exercise (https://ent.cochrane.org/ourevidence/prioritisation/scoping-projects). Cochrane Ear, Nose, \& Throat Disorders (Cochrane ENT) group this has developed suites of reviews with an "optimal, shared protocol with a well-designed and consistent set of outcome measures" (Cochrane ENT Group, 2019).

In this report we describe a comprehensive exercise used to prioritise systematic reviews of interventions for tinnitus conducted for the Cochrane ENT group.

Subjective tinnitus is described as the perception of sound in the absence of an external sound source (Jastreboff and Hazell, 2004). It is a symptom experienced by $10-30 \%$ of the adult population (McCormack et al., 2016). About $20 \%$ of people with tinnitus experience it as 
Sereda et al. Prioritising topics for systematic review

bothersome (McCormack et al., 2016). Problems associated with tinnitus include sleep

disturbances, hearing difficulties, difficulties with concentration, social isolation, anxiety, depression, and emotional difficulties such as irritation or stress (Davis and El Refaie, 2000).

It is estimated that the prevalence of tinnitus in those adults seeking medical help for hearing problems is as high as $85 \%$ (Axelsson and Ringdahl, 1989; Davis and El Refaie, 2000;

Meikle and Taylor-Walsh, 1984).

Tinnitus represents a major financial burden to the healthcare system. For example, in England there are approximately 0.75 million primary care consultations each year where the primary complaint is tinnitus (El-Shunnar et al., 2011) and the average cost to the National Health System of tinnitus treatment per year is estimated to be GB£750M. The estimated annual societal costs of tinnitus in the UK is GB£2.7 billion (Stockdale et al., 2017).

There is currently no gold standard treatment for tinnitus, rather, various management strategies are used or have been trialled. Those include education and information, soundbased interventions, psychology-based interventions, self-help interventions, relaxation therapy, pharmacology-based interventions, manual physical therapy, magnetic stimulation, electrical stimulation, complementary and alternative therapies, and combination of two or more approaches (complex interventions). Guidelines for the management of tinnitus have been developed in the USA and Europe (Cima et al., 2019; Fuller et al., 2017a). In the UK, there are commissioning guidelines for tinnitus services for adults (Department of Health, 2009), and clinical practice guidance for the assessment and management of tinnitus in children (British Society of Audiology, 2015) A Clinical Knowledge Summary has been produced by the National Institute for Health and Care Excellence (NICE) and two national guidelines are in development: the first by NICE; the second by the British Society of Audiology (BSA). NICE has published the scope of the guidelines that are in development (https://www.nice.org.uk/guidance/gid-ng10077/documents/final-scope) outlining which 


\section{Sereda et al. Prioritising topics for systematic review}

73 factors will and will not be considered by the guidelines. Effective guidelines can only be

74

75

76

77

78

79

80

81

82

83

84

85

86

87

88

89

90

91 Search strategy

92 In July 2017 we conducted a search of the Cochrane ENT Trials Register (via the Cochrane

93 Register of studies) for RCTs. There were no language, publication year, or publication status

94 restrictions. The search was run in the Cochrane ENT Register

\section{METHODS}

The prioritisation process was conducted in two stages. First, a scoping review was

conducted to estimate the volume of randomised controlled trial (RCT) level evidence available, to facilitate prioritisation, to aid in the design of a template protocol, and to estimate the work involved in conducting a suite of priority reviews. Secondly, interventions were prioritised for review according to a set of pre-defined criteria.

\section{Scoping review}

We followed the methodological framework of Arksey and O'Malley (2005). This consisted of: (1) identifying potentially relevant records; (2) selecting relevant records; (3) extracting data items; and (4) collating, summarising, and reporting the results. The PRISMA-ScR checklist (Tricco et al., 2018) guided reporting of the methods and results of the scoping review. 
Sereda et al. Prioritising topics for systematic review

95 (https://ent.cochrane.org/resources/searching-studies/cochrane-ent-trials-register) using the

96 following strategy:

971 MESH DESCRIPTOR Tinnitus EXPLODE ALL AND INREGISTER

982 tinnit* AND INREGISTER

3 \#1 OR \#2 AND INREGISTER,

100 where MESH DESCRIPTOR - Medical Subject Headings: The National Library of Medicine 101 controlled vocabulary thesaurus, INREGISTER - in the Cochrane ENT register, EXPLODE 102 ALL - search for selected subject heading (Tinnitus) and all of the subject headings in its 103 family.

104 The Cochrane ENT Register is populated using the methods described on the Cochrane ENT 105 website (https://ent.cochrane.org/resources/searching-studies/cochrane-ent-trials-register).

106 We also searched the Cochrane database of Systematic Reviews for all published reviews and 107 protocols for Cochrane reviews with 'tinnitus' in the title.

108 Selection of studies

109 Three authors (MS, DJH, DAH) independently screened all abstracts to determine eligibility 110 for inclusion in the scoping review. Records were carried forward for full screening if at least 111 one of the authors selected it. We considered multiple articles reporting the same trial 112 together as a single record. Disagreements were discussed between authors until a consensus 113 was reached. Records were considered for inclusion according to PICOS (Methley et al., 114 2014), as follows:

115 Population: Children and/or adults with subjective tinnitus

116 Intervention: All interventions for subjective tinnitus

117 Comparator: No intervention (e.g. waiting list), different intervention, placebo 
Sereda et al. Prioritising topics for systematic review

118 Outcome: Did not form an inclusion criterion

119 Study design: Randomised controlled trials only.

\section{Data extraction}

121 Data were extracted using a bespoke template form designed by the authors (MS and DJH),

122 piloted on a subset of records, and revised before formal data extraction was undertaken.

123 PICOS data were extracted (population, intervention, comparator, outcomes, and outcome

124 measures used, and study design). Two authors independently extracted the data.

125 For each intervention, we recorded whether there were existing RCTs, the number of RCTs,

126 and whether those RCTs were included or not in existing Cochrane reviews. In scoping the

127 literature, drug trials were catalogued (by DMcF) according to the World Health

128 Organization (WHO) Collaborating Centre for Drug Statistics Methodology Anatomical

129 Therapeutic Chemical (ATC) Classification System (https://www.whocc.no/atc ddd index/).

130 Methodological assessment of published Cochrane reviews

131 A list of published Cochrane systematic reviews and published Cochrane protocols was

132 populated. When judging whether an existing Cochrane systematic review required updating

133 or replacing, we considered the date of the most recent literature search of the review, and

134 whether ongoing studies were identified in those reviews. Both of these factors were used to

135 consider whether there was new research that may alter the estimates of effect, the quality of

136 the overall evidence, or the conclusions drawn in the published review. Other methodological

137 aspects of the systematic reviews were assessed including (1) whether a Preferred Reporting

138 Items for Systematic Reviews and Meta-Analyses (PRISMA) diagram was included; (2)

139 whether the latest risk of bias tool was used; (3) whether a 'Summary of Findings (SoF)'

140 table was included; (4) whether the 'Grading of Recommendations, Assessment, 
Sereda et al. Prioritising topics for systematic review

141 Development and Evaluation' (GRADE; https://gradepro.org/) tool was used (Schünemann et 142 al., 2013); (5) whether the assessed outcomes included measures of benefits and harms of the 143 intervention; and (6) whether the review included all of the methods sections currently 144 recommended by Cochrane (Higgins and Green, 2011).

\section{Prioritisation process}

146 Authors of this scoping review were experts in tinnitus (clinical researchers, a psychologist,

147 ENT surgeon, and an audiologist) or experts in Cochrane systematic review methodology. All 148 authors took part in agreeing the criteria that were used to prioritise reviews. Firstly a list of 149 criteria was populated including criteria formulated according to the remit from National 150 Institute for Health Research (NIHR) with additional criteria proposed by individual authors.

151 Secondly authors ranked these criteria in order of importance. Based on the ranking, four 152 primary and four secondary criteria were formulated.

153 Primary criteria were whether:

1541 . the intervention was available for tinnitus management within the National Health Service (NHS) When considering drug treatments for tinnitus, this included drugs that were used on-licence such as betahistine for Ménière's disease-associated tinnitus. It also included drugs used that have been recorded as being used offlicence as a primary tinnitus treatment (Langguth et al., 2009; Hall et al., 2011; McFerran et al., 2018). It did not include drugs used primarily for treating comorbid conditions.

2. the intervention was included in the NICE document, Guidelines scope. Tinnitus: assessment and management. (https://www.nice.org.uk/guidance/gidng10077/documents/final-scope). This document outlined the proposed contents of the forthcoming NICE Guideline. 
Sereda et al. Prioritising topics for systematic review

3. there was 'no recommendation' or disagreement in recommendations for an intervention within or between current management guidelines

4. existing Cochrane systematic reviews concluded there was a lack of evidence for an intervention, but additional evidence is now available or if there was no current Cochrane review.

Secondary criteria were whether:

5. the intervention was already prioritised by healthcare users and healthcare practitioners in the James Lind Alliance Priority Setting Partnership for tinnitus as a 'top 10 ' treatment uncertainty.

6. there were sufficient new RCTs for a new or updated review to be meaningful.

7. interventions were referred to in the tinnitus research network (TINNET) European clinical practice guideline.

8. there was evidence for variability in clinical practice, within or across countries.

All methodological considerations, and importance to key stakeholders were considered together in prioritising updated and new systematic reviews. For each of the interventions authors judged how many of the primary and secondary criteria were met. From this a list of high priority reviews was formulated.

\section{RESULTS}

\section{Summary of existing Cochrane reviews}

The Cochrane Library contained 10 existing Cochrane reviews on tinnitus: amplification with hearing aids (Hoare et al., 2014), anticonvulsant drugs (Hoekstra et al., 2011), antidepressant drugs (Baldo et al., 2012), Cognitive Behavioural Therapy (CBT) (Martinez-Devesa et al., 2010), Ginkgo biloba (Hilton et al., 2013), hyperbaric oxygen (for idiopathic sudden 
Sereda et al. Prioritising topics for systematic review

sensorineural hearing loss and tinnitus) (Bennett et al., 2012), repetitive Transcranial

Magnetic Stimulation (rTMS) (Meng et al., 2011), sound therapy (masking) (Hobson et al., 2012), Tinnitus Retraining Therapy (TRT) (Phillips and McFerran, 2010a), and zinc supplements (Person et al., 2016). A further eight protocols for systematic reviews had been published. Four were protocols for reviews in progress: CBT (Fuller et al., 2017b), glutamate receptor antagonists (Imsuwansri et al., 2016), melatonin (Ajayi et al., 2014), and neuromodulation (desynchronisation) (Hoare et al., 2015). In the review of TRT (Phillips and McFerran, 2010a), the literature search unearthed a number of studies that purported to be TRT but on inspection did not adhere to the strict protocol described by the developers of TRT (Jastreboff and Hazell, 2004). Many of these studies observed the underlying principles of TRT and its scientific rationale which is generally referred to as the neurophysiological model of tinnitus (Jastreboff, 1990). The authors of the TRT Cochrane review therefore proposed to write a separate review of these studies which they described as modified TRT. After discussion it was decided that a single review of both standard (unmodified) TRT and modified TRT would be more appropriate and a protocol for a review was published (Phillips and McFerran, 2010b). However, progress on this new review was suspended at the suggestion of Cochrane. Methods in this protocol were judged as needing updating. The other three published protocols (acupuncture (Li et al., 2016), low-level laser therapy (Peng et al., 2014), and an overview of systematic reviews of interventions (Maldonado Fernández et al., 2015) were withdrawn before the reviews were conducted or completed. Eight of the 10 published Cochrane reviews were assessed as having outdated methods by the Cochrane methodologist (EA). The review of zinc supplementation was judged as up-to-date and the methods robust (Person et al., 2016). The review of amplification with hearing aids was judged to have up-to-date methods such that the decision to update would depend on 
Sereda et al. Prioritising topics for systematic review

213

214 Cochrane reviews was between one and eight.

215

216

\section{New trials for potential inclusion in Cochrane reviews} data regarding PICOS and trial status were extracted.

\section{Education and information}

\section{Sound-based interventions}

whether additional RCTs were identified. The number of records included in each of the 10

Scoping searches identified 1080 records (Figure 1). Based on title/abstract screening 731 records were selected for full text screening by at least one author. A further 318 records were excluded that were duplicates $(n=127)$, out of scope $(n=11)$, not randomised $(n=86)$, conference abstracts with no results published $(n=70)$, or required translation for which we did not have the resources (Chinese, Japanese, Swedish, Spanish; n=15). Nine abstracts/full texts were not available. An additional 24 records were identified from lists of references of systematic reviews bringing the total number of records for full text screening and data charting to 437. Among those, 365 records were identified that were new (not covered in existing Cochrane reviews) RCTs with published results: PICOS data were extracted from those records. In addition, 51 unpublished registered randomised trials were identified and

\section{*** INSERT FIGURE 1 ABOUT HERE***}

Eight trials were identified that examined information or education.

Forty-three new trials of sound-based interventions were identified. The interventions trialled included: 1) Amplification only devices ( $\mathrm{n}=8)$; 2) Sound generator only devices (sometimes referred to as maskers; $\mathrm{n}=20$ ); 3 ) Combination devices (i.e. combined amplification and sound generators; $n=5)$; 4) Acoustic Coordinated Reset (CR) Neuromodulation ( $\mathrm{n}=3)$; 5) 
Sereda et al. Prioritising topics for systematic review

237 Phase-tailored sound treatment $(n=1) ; 6)$ Spectrally tailored sound treatment $(n=2)$; and 7)

238 Auditory training $(\mathrm{n}=4)$.

$239 \quad$ Psychology-based interventions

240 Thirty-nine new trials of psychology-based intervention were identified. Thirty-three of those

241 trialled CBT interventions and three trialled counselling. For the purpose of this scoping

242 review we included all studies using cognitive and/or behavioural approaches to treatment. It

243 is worth noting that there is a published protocol for a revision of the Cochrane review of

244 CBT for tinnitus (Fuller et al., 2017a). This review will examine all interventions for tinnitus

245 that include cognitive, and/or behavioural interventions. Those would include Acceptance

246 and Commitment Therapy (ACT) and Mindfulness-based therapies, described as different

247 'waves' of CBT.

248 Self-help interventions

249 One trial was identified that examined a self-help intervention, namely an online discussion 250 forum.

251 Relaxation therapy

252 Eighteen trials of relaxation therapy were identified including: Neurofeedback/Biofeedback 253 (n=8); Hypnosis/Hypnotherapy $(n=3) ; 3)$ Relaxation $(n=7)$.

\section{$254 \quad$ Pharmacology-based interventions}

255 One hundred and fifty-eight new trials of pharmacological interventions for tinnitus were

256 identified. They were classified in nine different categories based on the WHO ATC system:

257 1) Alimentary tract and metabolism $(n=12) ; 2)$ Blood and blood forming organs $(n=8) ; 3)$

258 Cardiovascular system $(n=20) ; 4)$ Genito-urinary system and sex hormones $(n=5) ; 5)$

259 Musculo-skeletal system $(\mathrm{n}=3)$; 6) Nervous system $(\mathrm{n}=83)$; 7) Respiratory system $(\mathrm{n}=1) ; 8)$ 
Sereda et al. Prioritising topics for systematic review

260 Systemic hormonal preparations, excluding sex hormones and insulins ( $\mathrm{n}=8)$; and 9) Various

$261(n=2)$. Thirteen trials of non-classified (i.e. experimental) medications were also identified.

\section{Manual physical therapy}

263 Five trials of manual physical therapy were identified including: 1) Cervical spine treatment

264 ( $\mathrm{n}=3)$; 2) Myofascial trigger point deactivation ( $\mathrm{n}=1)$; and 3) Temporomandibular Joint

265 Treatment $(\mathrm{n}=1)$.

266 Magnetic stimulation

267 Forty-one trials of magnetic stimulation were identified: 1) Repetitive Transcranial Magnetic

268 Stimulation (rTMS, n=36), 2) Continuous Theta Burst Stimulation (cTBS, n=2); 3) Deep

269 Transcranial Magnetic Stimulation ( $\mathrm{n}=1)$; 4) Electromagnetic Ear Stimulation ( $\mathrm{n}=1)$; and 5)

270 Rare-earth magnets placed close to the tympanic membrane $(n=1)$.

\section{$271 \quad$ Electrical stimulation}

272 Twenty-three new trials of electrical stimulation were identified including: 1) Cochlear

273 implant ( $\mathrm{n}=3) ; 2$ ) Transcranial Alternating Current Stimulation (tACS; $\mathrm{n}=1) ; 3$ ) Transcranial

274 Direct Current Stimulation (tDCS; $\mathrm{n}=11) ;$ 4) Vagus Nerve Stimulation (VNS; $\mathrm{n}=3$ ); 5)

275 Transcutaneous Electrical Nerve Stimulation (TENS; n=2); 6) Ear electrical stimulation via

276 surface tympanic electrode $(n=1)$; and 7) External electrical stimulation via mastoid bones

$277(\mathrm{n}=1)$. According to the published Cochrane protocol of neuromodulation

278 (desynchronisation) for tinnitus (Hoare et al., 2015), all trials of electrical stimulation for

279 tinnitus are likely to be included.

\section{Complementary and alternative therapies}

281 Fifty-six trials of complementary and alternative therapies were identified including: 1)

282 Acupuncture $(\mathrm{n}=26) ; 2)$ Dietary supplements and herbal remedies $(\mathrm{n}=10) ; 3)$ Laser treatment 
Sereda et al. Prioritising topics for systematic review

$283(\mathrm{n}=14) ; 4)$ Ozone $(\mathrm{n}=1) ; 5)$ Ultrasound $(\mathrm{n}=2) ; 6)$ Vibratory stimulation $(\mathrm{n}=2)$; and 7) Virtual 284 reality $(\mathrm{n}=1)$.

\section{Complex interventions}

286 Twenty-four trials of complex interventions were identified including: 1) Heidelberg Neuro-

287 Music Therapy $(n=2) ; 2)$ Perceptual/cognitive training $(n=4) ; 3)$ Progressive Tinnitus

288 Management (PTM, n=4); 4) Tinnitus Retraining Therapy (TRT, including modified TRT;

$289 \mathrm{n}=9)$; 5) Combination of psychological approaches with other management strategies $(\mathrm{n}=3)$;

290 6) bimodal treatment involving TRT with EMDR and TRT with CBT (n=1); and 7) a

291 combination of sound based, educational and integrated medicine therapies $(n=1)$.

\section{Priority reviews on tinnitus}

293 Three high priority reviews were identified based on the pre-defined priority criteria. Those 294 were: 1) sound therapy using amplification devices and/or sound generators for tinnitus; 2) 295 betahistine; 3) CBT.

296 Sound therapy met the first three primary priority criteria, the existing Cochrane reviews 297 concluded a lack of evidence of clinical effectiveness (Hoare et al., 2014a, Hobson et al., 298 2012) and new trials were identified. Our recommendation was that a priority Cochrane 299 review should include amplification only devices, combination devices (combined 300 amplification and sound generation), and sound generators. Suggested comparisons for 301 inclusion were: 1) Amplification only vs waiting-list control, placebo, education/information 302 only with no device; 2) Combination devices vs waiting-list control, placebo, 303 education/information only with no device, amplification only, sound generator only; 3 )

304 Sound generator only vs waiting-list control, placebo, education/information only with no 305 device. Trials that have conditions that explicitly included counselling (such as TRT, PTM, 306 Neuromonics) should be excluded. Counselling was defined according to Culley and Bond 
Sereda et al. Prioritising topics for systematic review

307 (2011) as a process that aims to empower patients to reach decisions and take actions for

308 themselves. Establishing a therapeutic relationship, clarifying and defining problems,

309 planning actions, and managing expectations are all key features of the approach. Education

310 and information giving can be entirely one-way, whereas counselling is about empowerment

311 and enabling patients to arrive at their own solutions using their own internal resources.

312 Therefore, unless there were explicit efforts and description of a process towards

313 empowerment in trial reports, and a trained therapist delivered it, then it was not considered

314 counselling.Betahistine also met the first three primary priority criteria and there is no

315 existing Cochrane review. We identified six trials for consideration. Comparisons should

316 include placebo, no intervention, education and information only. However, it should be

317 noted that only three trials include the above comparisons $(n=3)$ and the others would not be

318 suitable for synthesis. Subgroup analyses with and without Ménière's disease should also be

319 considered, but we note that there is an existing Cochrane review on Betahistine for

320 Ménière's disease or syndrome which has impact on tinnitus symptom severity as a

321 secondary outcome (Van Esch et al., 2018).

322 CBT met the first three primary priority criteria. Although there is an existing Cochrane

323 review (Martinez-Devesa et al., 2010) it is now outdated and does not include all cognitive,

324 and/or behavioural interventions (Acceptance and Commitment Therapy (ACT) and

325 Mindfulness-based therapies, described as different 'waves' of CBT). A Cochrane review

326 examining all cognitive and behavioural approaches for tinnitus is currently ongoing (Fuller

327 et al., 2017b).

328 Further priorities (meeting fewer priority criteria) included: 1) Gingko biloba; 2) anxiolytics;

329 3) hypnotics; 4) antiepileptics; 5) neuromodulation. 
Sereda et al. Prioritising topics for systematic review

330 Gingko biloba met the first two primary priority criteria. The existing Cochrane review

331 concluded a lack of evidence for effectiveness (Hilton et al., 2013) and new trials were

332 identified. Suggested comparisons include placebo, no intervention, education and

333 information only. Anxiolytics met the first two primary criteria and there is no existing

334 Cochrane review. Nine trials have been identified which may be eligible. Suggested

335 comparisons are placebo, no intervention, education and information only. Hypnotics meets

336 the first two primary criteria and there is no existing Cochrane review. Eight trials have been

337 identified which may be eligible for inclusion. Suggested comparisons are placebo, no

338 intervention, education and information only. Antiepileptics met the first two primary criteria

339 and there is no existing Cochrane review. Eleven trials have been identified. Suggested

340 comparisons include placebo, no intervention, education and information only.

341 Neuromodulation met two primary criteria including being in scope of the NICE guidelines.

342 However, a Cochrane review of neuromodulation for tinnitus is currently ongoing (Hoare et 343 al., 2015).

\section{CONCLUSIONS}

347 This technical report highlights a comprehensive exercise we undertook to prioritise topics of

348 unmet need for high-quality systematic review in tinnitus management.

349 Importantly, these priority reviews will respond to unanswered questions identified in current 350 and developing clinical practice guidelines for tinnitus. Three high priority reviews are

351 recommended: 1) sound therapy using amplification devices and/or sound generators for

352 tinnitus; 2) betahistine; 3) Cognitive Behaviour Therapy. Further priorities are: 4) Gingko

353 biloba; 5) anxiolytics; 6) hypnotics; 7) antiepileptics; 8) neuromodulation. 
Sereda et al. Prioritising topics for systematic review

354 Applying a prioritisation process ensures that resources are invested most effectively in work that meets the needs of funders and stakeholders and addresses known discrepancies or gaps in clinical knowledge. This particular prioritisation work focused on UK clinical practice for tinnitus and therefore the relevant priority criteria, such as availability of the intervention within the NHS and inclusion in the scope of the NICE tinnitus guideline. However, the process can easily be adapted to a range of international, national or local settings and priorities. For example, regional or country-specific clinical practice can be taken into

consideration as well as guidelines at the national, regional or international level (e.g.

362 European or country-specific) when formulating the priority criteria.

363 The scoping exercise described here has already resulted in the expedited production of two

364 Cochrane systematic reviews (Sereda et al., 2018; Wegner et al., 2018) in part to inform the

NICE guideline on tinnitus which is currently under development. A further three priority reviews are currently in progress (Fuller et al., 2017b; Hoare et al. 2015; and Gingko biloba 367 protocol in preparation).

\section{Acknowledgements}

MS, DB, IP, and DJH are funded by the National Institute for Health Research (NIHR)

Biomedical Research Centre programme. DAH is an NIHR Senior Investigator. The views expressed are those of the authors and not necessarily those of the NIHR, the NHS, or the

Department of Health and Social Care. RFFC is funded through The Netherlands

375 Organisation for Scientific Research (NWO); Innovational Research Incentives Scheme Veni. 
Sereda et al. Prioritising topics for systematic review

377

378

379

380

381

382

383

384

385

386

387

388

389

390

391

392

393

394

395

396

397

398

399

comments on the review process and the report. We would also like to thank Sandra Smith and Snigdha Dutta for their assistance in the manuscript preparation.

\section{References}

Ajayi, O. V., Phillips, J. S., Laopaiboon, M., McFerran, D. 2014. Melatonin for tinnitus. Cochrane Database of Systematic Reviews, 12: CD011435. https://doi.org/10.1002/14651858.CD011435

Arksey, H., O’Malley L. 2005. Scoping studies: towards a methodological framework. International Journal of Social Research Methodology: Theory \& Practice, 8: 19-32. https://doi.org/10.1080/1364557032000119616

Axelsson, A., Ringdahl, A. 1989. Tinnitus--a study of its prevalence and characteristics. British Journal of Audiology, 23(1): 53-62. https://doi.org/10.3109/03005368909077819

Baldo, P., Doree, C., Lazzarini, R., Molin, P., McFerran, D. 2012. Antidepressants for patients with tinnitus (Review). Cochrane Database of Systematic Reviews, 9: CD003853. https://doi.org/10.1002/14651858.CD003853.pub3

Bennett, M., Kertesz, T., Perleth, M., Yeung, P., Lehm, J., Lehm, J. P. 2012. Hyperbaric oxygen for idiopathic sudden sensorineural hearing loss and tinnitus (Review). Cochrane Database of Systematic Reviews, 10: CD004739. https://doi.org/10.1002/14651858.CD004739.pub4

British Society of Audiology. 2015. Tinnitus in Children Practice Guidance. Retrieved May 18, 2018, from http://www.thebsa.org.uk/now-available-new-bsa-tinnitus-in-childrenpractice-guidance/ 
Sereda et al. Prioritising topics for systematic review

400 Cima, R.F.F., Mazurek, B., Haider, H., Kikidis, D., Lapira, A., Noreña, A., Hoare, D.J. 2019. 401 A multidisciplinary guideline for tinnitus: diagnostics, assessment, and treatment. $H N O$, 40267 (Suppl 1): 10-42. https://doi.org/10.1007/s00106-019-0633-7

403 Cochrane ENT group. 2019. Scoping projects. Retrieved July 24, 2019, from https://ent.cochrane.org/our-evidence/prioritisation/scoping-projects.

405 Culley, S., Bond, T. 2011. Integrative counselling skills in action. Sage Publications Ltd; 3rd 406 edition.

407 Davis, A., \& El Refaie, A. 2000. Epidemiology of tinnitus. In R. Tyler (Ed.), Tinnitus 408 Handbook. San Diego: Singular Publishing Group.

409 Department of Health. 2009. Provision of Services for Adults with Tinnitus: A Good Practice $410 \quad$ Guide. https://doi.org/http://webarchive.nationalarchives.gov.uk/20130107105354/http:/www.d h.gov.uk/prod consum dh/groups/dh_digitalassets/documents/digitalasset/dh_093810.p $\underline{\mathrm{df}}$

El-Shunnar, S. K., Hoare, D. J., Smith, S., Gander, P. E., Kang, S., Fackrell, K., and Hall, D. A. 2011. Primary care for tinnitus: Practice and opinion among GPs in England. Journal of Evaluation in Clinical Practice, 17(4): 684-692. https://doi.org/10.1111/j.1365-

Fuller, T.E., Haider, H.F., Kikidis, D., Lapira, A., Mazurek, B., Norena, A., Rabau, S., Lardinois, R., Cederroth, C.R., Edvall, N.K., Brueggemann, P.G., Rosing, S.N., 2753.2011.01696.x Kapandais, A., Lungaard, D., Hoare, D.J., Cima, R.F. 2017a. Different Teams, Same Conclusions? A Systematic Review of Existing Clinical Guidelines for the Assessment and Treatment of Tinnitus in Adults. Frontiers in Psychology, 8:206. https://doi: 10.3389/fpsyg.2017.00206. eCollection 2017. 
Sereda et al. Prioritising topics for systematic review

424 Fuller, T.E., Cima, R., Langguth, B., Mazurek, B., Waddell, A., Hoare, D. J., and Vlaeyen, J.

W. S. 2017b. Cognitive behavioural therapy for tinnitus. Cochrane Database of

Systematic Reviews, 4: CD012614. https://doi.org/10.1002/14651858.CD012614

Greenwell, K., Sereda, M., Coulson, N, El Refaie, A., Hoare, D.J. 2016. A systematic review

of techniques and effects of self-help interventions for tinnitus: Application of

taxonomies from health psychology. Int J Audiol, Suppl 3: S79-89.

https://doi.org/10.3109/14992027.2015.1137363

Hall, D.A., Láinez, M.J., Newman, C.W., Sanchez, T.G., Egler, M., Tennigkeit, F., Koch, M., Langguth, B. 2011. Treatment options for subjective tinnitus: self reports from a sample

Services Research, 11: 302. https://doi.org/10.1186/1472-6963-11-302

Higgins, J.P.T. and Green, S. 2011. Cochrane Handbook for Systematic Reviews of Interventions Version 5.1.0 [updated March 2011]. The Cochrane Collaboration. Retrieved May 18, 2018, from www.cochrane-handbook.org

Hilton, M., Zimmermann, E.F, Stuart, E. 2013. Ginkgo biloba for tinnitus. Cochrane Database of Systematic Reviews, 3: CD003852. https://doi.org/10.1002/14651858.CD003852

Hoare, D. J., Edmondson-Jones, M., Sereda, M., Akeroyd, M. A., and Hall, D. 2014. Amplification with hearing aids for patients with tinnitus and co-existing hearing loss (Review). Cochrane Database of Systematic Reviews, 1: CD010151.

Hoare, D. J., Whitham, D., Henry, J. A., and Shorter, G. W. 2015. Neuromodulation 
Sereda et al. Prioritising topics for systematic review

448 Hobson, J., Chisholm, E. J., and Loveland, M. E. 2012. Sound therapy (masking) in the

449 management of tinnitus in adults. Cochrane Database of Systematic Reviews, 11:

$450 \quad$ CD006371. https://doi.org/10.1002/14651858.CD006371

451 Hoekstra, C. E. L., Rynja, S. P., Van Zanten, G. A., and Rovers, M. 2011. Anticonvulsants

452 for tinnitus. Cochrane Database of Systematic Reviews, 7: CD007960.

453 https://doi.org/10.1002/14651858.CD007960

454 Imsuwansri, T., Hoare, D. J., Phaisaltuntiwongs, W., Srisubat, A., and Snidvongs, K. 2016.

455 Glutamate receptor antagonists for tinnitus ( Protocol). Cochrane Database of

456 Systematic Reviews, 10: CD012391. https://doi.org/10.1002/14651858.CD012391

457 Jastreboff, P.J. 1990. Phantom auditory perception (tinnitus): mechanisms of generation and

458 perception. Neuroscience Research, 8(4): 221-54. https://doi.org/10.1016/0168-

$459 \quad \underline{0102(90) 90031-9}$

460 Jastreboff, P. J., and Hazell, J. W. P. 2004. Tinnitus Retraining Therapy. Cambridge

$461 \quad$ University Press. https://doi.org/10.1017/CBO9780511544989

462 Langguth, B., Salvi, R., Elgoyhen, A.B. 2009. Emerging pharmacotherapy of tinnitus. Expert 463 Opinion on Emerging Drugs, 14(4): 687-702.

$464 \quad$ https://doi.org/10.1517/14728210903206975

465 Li, Y., Zeng, R. F., and Zheng, D. 2016. Acupuncture for tinnitus. Cochrane Database of 466 Systematic Reviews, 11: CD008149. https://doi.org/10.1002/14651858.CD008149.pub2

467 Maldonado Fernández, M., Shin, J., Scherer, R. W., and Murdin, L. 2015. Interventions for 468 tinnitus in adults: an overview of systematic reviews. Cochrane Database of Systematic 469 Reviews, 1: CD011795. https://doi.org/10.1002/14651858.CD011795

470 Martinez-Devesa, P., Perera, R., Theodoulou, M., and Waddell, A. 2010. Cognitive 
Sereda et al. Prioritising topics for systematic review

471

472

473

474

475

476

477

478

479

480

481

482

483

484

485

486

487

488

489

490

491

492

493

behavioural therapy for tinnitus. Cochrane Database of Systematic Reviews, 9:

CD005233. https://doi.org/10.1002/14651858.CD005233.pub3

McCormack, A., Edmondson-Jones, M., Somerset, S., and Hall, D. 2016. A systematic review of the reporting of tinnitus prevalence and severity. Hearing Research, 337: 7079. https://doi.org/10.1016/j.heares.2016.05.009

McFerran, D., Hoare, D.J., Carr, S., Ray, J., Stockdale, D. 2018. Tinnitus services in the United Kingdom: a survey of patient experiences. BMC Health Services Research, 18(1): 110. https://doi.org/110.1186/s12913-018-2914-3.

Meikle, M., Taylor-Walsh, E. 1984. Characteristics of tinnitus and related observations in over 1800 tinnitus clinic patients. The Journal of Laryngology \& Otology, 9: 17-21. https://doi.org/10.1017/S1755146300090053

Meng, Z., Liu, S., Zheng, Y., and Phillips, J. S. 2011. Repetitive transcranial magnetic stimulation for tinnitus. Cochrane Database of Systematic Reviews, 10: CD007946. https://doi.org/10.1002/14651858.CD007946.pub2

Methley, A.M., Campbell, S., Chew-Graham, C., McNally, R., Cheraghi-Sohi, S. 2014. PICO, PICOS and SPIDER: a comparison study of specificity and sensitivity in three search tools for qualitative systematic reviews. BMC Health Services Research, 14:579. https://doi.org/10.1186/s12913-014-0579-0

Morata, T.C., Hickson, L., Wong, L. 2017. The IJA system for systematic reviews: "the whys and hows". Int J Audiol, 56(4): 213-214. https://doi.org/10.1080/14992027.2016.1275044

Peng, Z., Chen, X., Gong, S., and Chen, C. 2012. Low-level laser therapy for tinnitus. Cochrane Database of Systematic Reviews, 9: CD009811 
Sereda et al. Prioritising topics for systematic review

494

495

496

497

498

499

500

501

502

503

504

505

506

507

508

509

510

511

512

513

514

515

516

https://doi.org/10.1002/14651858.CD009811

Person, O. C., Puga, M. E. S., and da Silva, E. M. K. 2016. Zinc supplementation for tinnitus (Review). Cochrane Database of Systematic Reviews, 11: CD009832. https://doi.org/10.1002/14651858.CD009832

Phillips, J. S., and McFerran, D. 2010a. Tinnitus retraining therapy (TRT) for tinnitus patients. Cochrane Database of Systematic Reviews, 3: CD007330. https://doi.org/10.1002/14651858.CD007330

Phillips, J. S., and McFerran, D. 2010b. Neurophysiological model-based treatments for tinnitus. Cochrane Database of Systematic Reviews, 1: CD008248. https:// 10.1002/14651858.CD008248.pub2

Schünemann, H., Brożek, J., Guyatt, G., and Oxman A (editors). 2013. The GRADE Working Group. GRADE Handbook for Grading Quality of Evidence and Strength of Recommendations [Updated October 2013]. Retrieved May 18, 2018, from http://gdt.guidelinedevelopment.org/app/handbook/handbook.html

Sereda, M., Xia, J., E1 Refaie, A., Hall, D.A., Hoare, D.J. 2018. Sound therapy (using amplification devices and/or sound generators) for tinnitus. Cochrane Database of Systematic Reviews, 12: CD013094. https://doi.org/10.1002/14651858.CD013094.pub2

Stockdale, D., McFerran, D., Brazier, P., Pritchard, C., Kay, T., Dowrick, C., Hoare, D. J. 2017. An economic evaluation of the healthcare cost of tinnitus management in the UK. BMC Health Services Research, 17(1): 1-9. https://doi.org/10.1186/s12913-017-2527-2

Tricco, A.C., Lillie, E., Zarin, W., O'Brien, K.K., Colquhoun, H., Levac, D., Moher, D., Peters, M.D.J., Horsley, T., Weeks, L., Hempel, S., Akl, E.A., Chang, C., McGowan, J., Stewart, L., Hartling, L., Aldcroft, A., Wilson, M.G., Garritty, C., Lewin, S., Godfrey, 
Sereda et al. Prioritising topics for systematic review

517

518

519

520

521

522

523

524

525

526

527

528

529

530

531

532

533

534

535

536

van Esch, B., van der Zaag-Loonen, H. J., Bruintjes, T., Murdin, L., James, A., van Benthem, P. P. 2018. Betahistine for Ménière's disease or syndrome. Cochrane Database of Systematic Reviews, 1: CD012914. https://doi: 10.1002/14651858.CD012914

Wegner, I., Hall, D.A., Smit, A.L., McFerran, D., Stegeman, I. 2018. Betahistine for tinnitus. Cochrane Database of Systematic Reviews, 12: CD013093.

https://doi.org/14651858.CD013093.pub2

\section{FIGURE LEGEND}

Figure 1. Flow diagram illustrating search strategy and scoping review stages

\section{SUPPLEMENTAL MATERIAL}

Supplemental material 1. Summary of priority criteria for each of the interventions 
Sereda et al. Prioritising topics for systematic review

\section{ABSTRACT}

2 Objective: To develop an innovative prioritisation process to identify topics for new or

3 updated systematic reviews of tinnitus and hearing research.

4 Design: A two stage prioritisation process was devised. Firstly, a scoping review assessed the

5 amount of randomised-controlled-trial-level evidence available. This enabled development of

6 selection criteria for future reviews, aided the design of template protocol, and suggested the

7 scale of work that would be required to conduct these reviews. Secondly, using the pre-

8 defined primary and secondary criteria, interventions were prioritised for systematic review.

$9 \quad$ Study sample: Searches identified 1080 records. After removal of duplicates and out of

10 scope works, 437 records remained for full data charting.

11 Results: The process was tested, using subjective tinnitus as the clinical condition and using

12 Cochrane as the systematic review platform. The criteria produced by this process identified

13 three high priority reviews: 1) Sound therapy using amplification devices and/or sound

14 generators; 2) Betahistine, and 3) Cognitive Behaviour Therapy. Further secondary priorities

15 were: 4) Gingko biloba, 5) Anxiolytics, 6) Hypnotics, 7) Antiepileptics, and 8)

16 Neuromodulation.

17 Conclusions: A process was developed which successfully identified priority areas for

18 Cochrane systematic reviews of interventions for subjective tinnitus. This technique could

19 easily be transferred to other conditions and other types of systematic reviews.

22 Keywords: Cochrane, systematic review, priority, management, treatment, tinnitus 
Sereda et al. Prioritising topics for systematic review

\section{INTRODUCTION}

Systematic reviews and meta-analyses represent the highest level of evidence for the effectiveness of clinical interventions and hold a critical place in informing health policy and evidence-based practice (Greenwell et al.2016; Morata et al., 2017). One of the foremost organisations producing systematic reviews is Cochrane, which is a UK based charity (notfor-profit organisation) that supervises a global independent network of healthcare practitioners, researchers, patient advocates and others. It represents more than 11,000 members and over 68,000 supporters from over 130 countries (https://www.cochrane.org/about-us). Cochrane authors conduct systematic reviews of health-care interventions and diagnostic tests which are published as Cochrane Reviews in the Cochrane Library. Previously, Cochrane authors self-selected topics for their reviews and submitted proposals to Cochrane for approval. This process has been updated and now, Cochrane groups are encouraged to work strategically to respond to the needs of funders and key stakeholders to produce reviews on topics of the highest priority to users. One approach to prioritising these reviews is to conduct a scoping exercise (https://ent.cochrane.org/ourevidence/prioritisation/scoping-projects). Cochrane Ear, Nose, \& Throat Disorders (Cochrane ENT) group this has developed suites of reviews with an "optimal, shared protocol with a well-designed and consistent set of outcome measures" (Cochrane ENT Group, 2019).

In this report we describe a comprehensive exercise used to prioritise systematic reviews of interventions for tinnitus conducted for the Cochrane ENT group.

Subjective tinnitus is described as the perception of sound in the absence of an external sound source (Jastreboff and Hazell, 2004). It is a symptom experienced by $10-30 \%$ of the adult population (McCormack et al., 2016). About $20 \%$ of people with tinnitus experience it as 
Sereda et al. Prioritising topics for systematic review

bothersome and negatively affecting quality of life-(McCormack et al., 2016). Problems associated with tinnitus include sleep disturbances, hearing difficulties, difficulties with concentration, social isolation, anxiety, depression, and emotional difficulties such as irritation or stress (Davis and El Refaie, 2000). It is estimated that the prevalence of tinnitus in those adults seeking medical help for hearing problems is as high as $85 \%$ (Axelsson and Ringdahl, 1989; Davis and El Refaie, 2000; Meikle and Taylor-Walsh, 1984).

Tinnitus represents a major financial burden to the healthcare system. For example, in England there are approximately 0.75 million primary care consultations each year where the primary complaint is tinnitus (El-Shunnar et al., 2011) and the average cost to the National Health System of tinnitus treatment per year is estimated to be GB£750M. The estimated annual societal costs of tinnitus in the UK is GB£2.7 billion (Stockdale et al., 2017).

There is currently no gold standard treatment for tinnitus, rather, various management strategies are used or have been trialled. Those include education and information, soundbased interventions, psychology-based interventions, self-help interventions, relaxation therapy, pharmacology-based interventions, manual physical therapy, magnetic stimulation, electrical stimulation, complementary and alternative therapies, and combination of two or more approaches (complex interventions). Guidelines for the management of tinnitus have been developed in the USA and Europe (Cima et al., 2019; Fuller et al., 2017a). In the UK, there are commissioning guidelines for tinnitus services for adults (Department of Health, 2009), and clinical practice guidance for the assessment and management of tinnitus in children (British Society of Audiology, 2015) A Clinical Knowledge Summary has been produced by the National Institute for Health and Care Excellence (NICE) and two national guidelines are in development: the first by NICE; the second by the British Society of Audiology (BSA). NICE has published the scope of the guidelines that are in development (https://www.nice.org.uk/guidance/gid-ng10077/documents/final-scope) outlining which 
Sereda et al. Prioritising topics for systematic review

73 factors will and will not be considered by the guidelines. Effective guidelines can only be developed if there is strong evidence-based information available. If such high-level evidence is not available, recommendations arising from the guidelines are weak and clinically ineffective. These are just some of the drivers for prioritising new and updating existing

77 Cochrane systematic reviews of interventions for tinnitus.

\section{METHODS}

The prioritisation process was conducted in two stages. First, a scoping review was

conducted to estimate the volume of randomised controlled trial (RCT) level evidence available, to facilitate prioritisation, to aid in the design of a template protocol, and to estimate the work involved in conducting a suite of priority reviews. Secondly, interventions were prioritised for review according to a set of pre-defined criteria.

\section{Scoping review}

86 We followed the methodological framework of Arksey and O’Malley (2005). This consisted

87 of: (1) identifying potentially relevant records; (2) selecting relevant records; (3) extracting

88 data items; and (4) collating, summarising, and reporting the results. The PRISMA-ScR

89 checklist (Tricco et al., 2018) guided reporting of the methods and results of the scoping 90 review.

\section{$91 \quad$ Search strategy}

92 In July 2017 we conducted a search of the Cochrane ENT Trials Register (via the Cochrane 93 Register of studies) for RCTs. There were no language, publication year, or publication status 94 restrictions. The search was run in the Cochrane ENT Register 
Sereda et al. Prioritising topics for systematic review

95 (https://ent.cochrane.org/resources/searching-studies/cochrane-ent-trials-register) using the

96 following strategy:

971 MESH DESCRIPTOR Tinnitus EXPLODE ALL AND INREGISTER

982 tinnit* AND INREGISTER

\section{3 \#1 OR \#2 AND INREGISTER,}

where MESH DESCRIPTOR - Medical Subject Headings: The National Library of Medicine controlled vocabulary thesaurus, INREGISTER - in the Cochrane ENT register, EXPLODE ALL - search for selected subject heading (Tinnitus) and all of the subject headings in its family.

The Cochrane ENT Register is populated using the methods described on the Cochrane ENT website (https://ent.cochrane.org/resources/searching-studies/cochrane-ent-trials-register).

We also searched the Cochrane database of Systematic Reviews for all published reviews and protocols for Cochrane reviews with 'tinnitus' in the title.

\section{Selection of studies}

Three authors (MS, DJH, DAH) independently screened all abstracts to determine eligibility for inclusion in the scoping review. Records were carried forward for full screening if at least one of the authors selected it. We considered multiple articles reporting the same trial together as a single record. Disagreements were discussed between authors until a consensus was reached. Records were considered for inclusion according to PICOS (Methley et al., 2014), as follows:

Population: Children and/or adults with subjective tinnitus

Intervention: All interventions for subjective tinnitus 
Sereda et al. Prioritising topics for systematic review

118 Outcome: Did not form an inclusion criterion

119 Study design: Randomised controlled trials only.

\section{Data extraction}

121 Data were extracted using a bespoke template form designed by the authors (MS and DJH),

122 piloted on a subset of records, and revised before formal data extraction was undertaken.

123 PICOS data were extracted (population, intervention, comparator, outcomes, and outcome

124 measures used, and study design). Two authors independently extracted the data.

125 For each intervention, we recorded whether there were existing RCTs, the number of RCTs,

126 and whether those RCTs were included or not in existing Cochrane reviews. In scoping the

127 literature, drug trials were catalogued (by DMcF) according to the World Health

128 Organization (WHO) Collaborating Centre for Drug Statistics Methodology Anatomical

129 Therapeutic Chemical (ATC) Classification System (https://www.whocc.no/atc_ddd_index/).

130 Methodological assessment of published Cochrane reviews

131 A list of published Cochrane systematic reviews and published Cochrane protocols was

132 populated. When judging whether an existing Cochrane systematic review required updating

133 or replacing, we considered the date of the most recent literature search of the review, and

134 whether ongoing studies were identified in those reviews. Both of these factors were used to

135 consider whether there was new research that may alter the estimates of effect, the quality of

136 the overall evidence, or the conclusions drawn in the published review. Other methodological

137 aspects of the systematic reviews were assessed including (1) whether a Preferred Reporting

138 Items for Systematic Reviews and Meta-Analyses (PRISMA) diagram was included; (2)

139 whether the latest risk of bias tool was used; (3) whether a 'Summary of Findings (SoF)'

140 table was included; (4) whether the 'Grading of Recommendations, Assessment, 
Sereda et al. Prioritising topics for systematic review

141 Development and Evaluation' (GRADE; https://gradepro.org/) tool was used (Schünemann et 142 al., 2013); (5) whether the assessed outcomes included measures of benefits and harms of the 143 intervention; and (6) whether the review included all of the methods sections currently 144 recommended by Cochrane (Higgins and Green, 2011).

\section{Prioritisation process}

146 Authors of this scoping review were experts in tinnitus (clinical researchers, a psychologist, 147 ENT surgeon, and an audiologist) or experts in Cochrane systematic review 148 methodology. Authors of this scoping review were experts in tinnitus, clinieal researchers, at psychologist, ENT surgeon, and an audiologist or experts in Cochrane systematic review methodology. All authors took part in agreeing the criteria that were used to prioritise reviews. Firstly a list of criteria was populated including criteria formulated according to the remit from National Institute for Health Research (NIHR) with additional criteria proposed by individual authors. Secondly authors ranked these criteria in order of importance. Based on the ranking, four primary and four secondary criteria were formulated.

Primary criteria were whether:

1. the intervention is was available for tinnitus management within the National Health Service (NHS) When considering drug treatments for tinnitus, this included drugs that are-were used on-licence such as betahistine for Ménière's disease-associated tinnitus. It also included drugs used that have been recorded as being used offlicence as a primary tinnitus treatment (Langguth et al., 2009; Hall et al., 2011; McFerran et al., 2018). It did not include drugs used primarily for treating comorbid conditions.

2. the intervention is was included in the NICE document, Guidelines scope. Tinnitus: assessment and management. (https://www.nice.org.uk/guidance/gid- 
Sereda et al. Prioritising topics for systematic review

165

ng10077/documents/final-scope). This document outlines-outlined the proposed contents of the forthcoming NICE Guideline.

3. there was 'no recommendation' or disagreement in recommendations for an intervention within or between current management guidelines

4. existing Cochrane systematic reviews concluded there was a lack of evidence for an intervention, but additional evidence is now available or if there wasis no current Cochrane review.

Secondary criteria were whether:

5. the intervention had was already been-prioritised by healthcare users and healthcare practitioners in the James Lind Alliance Priority Setting Partnership for tinnitus as a 'top 10' treatment uncertainty.

6. there were sufficient new RCTs for a new or updated review to be meaningful.

7. interventions were referred to in the tinnitus research network (TINNET) European clinical practice guideline.

8. there was evidence for variability in clinical practice, within or across countries.

All methodological considerations, and importance to key stakeholders were considered together in prioritising updated and new systematic reviews. For each of the interventions authors judged how many of the primary and secondary criteria were met. From this a list of high priority reviews was formulated.

\section{RESULTS}

\section{Summary of existing Cochrane reviews}

$\underline{\text { The Cochrane Library contained } 10 \text { existing Cochrane reviews on tinnitus: amplification with }}$ hearing aids (Hoare et al., 2014), anticonvulsant drugs (Hoekstra et al., 2011), antidepressant 


\section{Sereda et al. Prioritising topics for systematic review}

189 drugs (Baldo et al., 2012), Cognitive Behavioural Therapy (CBT) (Martinez-Devesa et al.,

2010), Ginkgo biloba (Hilton et al., 2013), hyperbaric oxygen (for idiopathic sudden

sensorineural hearing loss and tinnitus) (Bennett et al., 2012), repetitive Transcranial

Magnetic Stimulation (rTMS) (Meng et al., 2011), sound therapy (masking) (Hobson et al.,

193 2012), Tinnitus Retraining Therapy (TRT) (Phillips and McFerran, 2010a), and zinc

194 supplements (Person et al., 2016). A further eight protocols for systematic reviews had been published. Four were protocols for reviews in progress: CBT (Fuller et al., 2017b), glutamate

receptor antagonists (Imsuwansri et al., 2016), melatonin (Ajayi et al., 2014), and

197 neuromodulation (desynchronisation) (Hoare et al., 2015). In the review of TRT (Phillips and

198 McFerran, 2010a), the literature search unearthed a number of studies that purported to be

199 TRT but on inspection did not adhere to the strict protocol described by the developers of

200 TRT (Jastreboff and Hazell, 2004). Many of these studies observed the underlying principles

201 of TRT and its scientific rationale which is generally referred to as the neurophysiological

202 model of tinnitus (Jastreboff, 1990). The authors of the TRT Cochrane review therefore

203 proposed to write a separate review of these studies which they described as modified TRT.

204 After discussion it was decided that a single review of both standard (unmodified) TRT and

205 modified TRT would be more appropriate and a protocol for a review was published (Phillips

206 and McFerran, 2010b). However, progress on this new review was suspended at the

207 suggestion of Cochrane. Methods in this protocol were judged as needing updating. The other

208 three published protocols (acupuncture (Li et al., 2016), low-level laser therapy (Peng et al.,

209 2014), and an overview of systematic reviews of interventions (Maldonado Fernández et al.,

210 2015)) were withdrawn before the reviews were conducted or completed. There were 10

211 existing Cochrane reviews on tinnitus (Baldo et al., 2012; Bennett et al., 2012; Hilton et al.,

212 2013; Hoare et al., 2014; Hobson et al., 2012; Hoekstra et al., 2011; Martinez-Devesa et al.,

213 2010; Meng et al., 2011; Person et al., 2016; Phillips and McFerran, 2010a) published in The 
Sereda et al. Prioritising topics for systematic review

214 Cochrane Library. The interventions evaluated were Tinnitus Retraining Therapy (TRT),

215 Cognitive Behavioural Therapy (CBT), anticonvulsants, repetitive Transeranial Magnetic

216 Stimulation (rTMS), antidepressants, sound therapy (masking), Ginkgo biloba, hyperbaric

217 oxygen (for idiopathic sudden sensorineural hearing loss and tinnitus), zinc supplements, and 218 amplification with hearing aids. A further eight protocols for systematic reviews had been

219 published. Five were protocols for reviews in progress, on neuromodulation

220 (desynchronisation) (Hoare et al., 2015), neurophysiological model-based treatments (Phillips

221 and McFerran, 2010b), CBT (Fuller et al., 2017b), glutamate receptor antagonists

222 (Imsuwansri et al., 2016), and melatonin (Ajayi et al., 2014). The other three published

223 protocols (acupuncture, low-level laser therapy, and an overview of systematic reviews of

224 interventions) were withdrawn before the reviews were conducted or completed (Li et al.,

225 2016; Maldonado Fernández et al., 2015; Peng et al., 2014). The protocol for

226 neurophysiological based treatments for tinnitus (Phillips and McFerran, 2010b) planned to

227 include unmodified and modified TRT, meaning it would constitute an update to the TRT

228 review. However, progress on this new review has been suspended at the suggestion of

229 Cochrane. Methods in this protocol were judged as needing updating.

230 Eight of the 10 published Cochrane reviews were assessed as having outdated methods by the

231 Cochrane methodologist (EA). The review of zinc supplementation was judged as up-to-date

232 and the methods robust (Person et al., 2016). The review of amplification with hearing aids

233 was judged to have up-to-date methods such that the decision to update would depend on

234 whether additional RCTs were identified. The number of records included in each of the 10

235 Cochrane reviews was between one and eight.

236

237 New trials for potential inclusion in Cochrane reviews 
Sereda et al. Prioritising topics for systematic review

238 Scoping searches identified 1080 records (Figure 1). Based on title/abstract screening 731

239 records were selected for full text screening by at least one author. A further 318 records

240 were excluded that were duplicates $(n=127)$, out of scope $(n=11)$, not randomised $(n=86)$,

241 conference abstracts with no results published $(n=70)$, or required translation for which we

242 did not have the resources (Chinese, Japanese, Swedish, Spanish; $n=15$ ). Nine abstracts/full

243 texts were not available. An additional 24 records were identified from lists of references of

244 systematic reviews bringing the total number of records for full text screening and data

245 charting to 437. Among those, 365 records were identified that were new (not covered in

246 existing Cochrane reviews) RCTs with published results: PICOS data were extracted from

247 those records. In addition, 51 unpublished registered randomised trials were identified and 248 data regarding PICOS and trial status were extracted.

\section{Education and information}

253 Eight trials were identified that examined information or education.

\section{Sound-based interventions}

255 Forty-three new trials of sound-based interventions were identified. The interventions trialled 256 included: 1) Amplification only devices $(n=8)$; 2) Sound generator only devices (sometimes 257 referred to as maskers; $\mathrm{n}=20$ ); 3) Combination devices (i.e. combined amplification and 258 sound generators; $n=5)$; 4) Acoustic Coordinated Reset (CR) Neuromodulation $(n=3) ; 5)$

259 Phase-tailored sound treatment $(n=1) ; 6)$ Spectrally tailored sound treatment $(n=2)$; and 7)

260 Auditory training $(\mathrm{n}=4)$.

\section{Psychology-based interventions}


Sereda et al. Prioritising topics for systematic review

262 Thirty-nine new trials of psychology-based intervention were identified. Thirty-three of those

263 trialled CBT interventions and three trialled counselling. For the purpose of this scoping

264 review we included all studies using cognitive and/or behavioural approaches to treatment. It

265 is worth noting that there is a published protocol for a revision of the Cochrane review of

266 CBT for tinnitus (Fuller et al., 2017a). This review will examine all interventions for tinnitus

267 that include cognitive, and/or behavioural interventions. Those would include Acceptance

268 and Commitment Therapy (ACT) and Mindfulness-based therapies, described as different

269 'waves' of CBT.

\section{Self-help interventions}

271 One trial was identified that examined a self-help intervention, namely an online discussion 272 forum.

\section{Relaxation therapy}

274 Eighteen trials of relaxation therapy were identified including: Neurofeedback/Biofeedback 275 (n=8); Hypnosis/Hypnotherapy (n=3); 3) Relaxation $(n=7)$.

\section{Pharmacology-based interventions}

277 One hundred and fifty-eight new trials of pharmacological interventions for tinnitus were

278 identified. They were classified in nine different categories based on the WHO ATC system:

279 1) Alimentary tract and metabolism ( $n=12) ; 2)$ Blood and blood forming organs $(n=8) ; 3)$

280 Cardiovascular system $(n=20) ; 4)$ Genito-urinary system and sex hormones $(n=5) ; 5)$

281 Musculo-skeletal system (n=3); 6) Nervous system ( $\mathrm{n}=83)$; 7) Respiratory system $(\mathrm{n}=1) ; 8)$

282 Systemic hormonal preparations, excluding sex hormones and insulins ( $\mathrm{n}=8)$; and 9) Various

$283(\mathrm{n}=2)$. Thirteen trials of non-classified (i.e. experimental) medications were also identified. 
Sereda et al. Prioritising topics for systematic review

285 Five trials of manual physical therapy were identified including: 1) Cervical spine treatment

286 ( $\mathrm{n}=3)$; 2) Myofascial trigger point deactivation ( $\mathrm{n}=1)$; and 3) Temporomandibular Joint

287 Treatment $(\mathrm{n}=1)$.

288 Magnetic stimulation

289 Forty-one trials of magnetic stimulation were identified: 1) Repetitive Transcranial Magnetic 290 Stimulation (rTMS, n=36), 2) Continuous Theta Burst Stimulation (cTBS, n=2); 3) Deep

291 Transcranial Magnetic Stimulation $(n=1)$; 4) Electromagnetic Ear Stimulation ( $\mathrm{n}=1)$; and 5)

292 Rare-earth magnets placed close to the tympanic membrane $(n=1)$.

293

294

295

296

297

298

299

300

301

302

303

304

305

306

307

\section{Electrical stimulation}

Twenty-three new trials of electrical stimulation were identified including: 1) Cochlear implant ( $\mathrm{n}=3$ ); 2) Transcranial Alternating Current Stimulation (tACS; $\mathrm{n}=1$ ); 3) Transcranial Direct Current Stimulation (tDCS; $n=11) ; 4$ ) Vagus Nerve Stimulation (VNS; $n=3) ; 5$ ) Transcutaneous Electrical Nerve Stimulation (TENS; n=2); 6) Ear electrical stimulation via surface tympanic electrode ( $\mathrm{n}=1)$; and 7) External electrical stimulation via mastoid bones $(n=1)$. According to the published Cochrane protocol of neuromodulation (desynchronisation) for tinnitus (Hoare et al., 2015), all trials of electrical stimulation for tinnitus are likely to be included.

\section{Complementary and alternative therapies}

Fifty-six trials of complementary and alternative therapies were identified including: 1) Acupuncture $(n=26) ; 2)$ Dietary supplements and herbal remedies $(n=10) ; 3)$ Laser treatment $(\mathrm{n}=14) ; 4)$ Ozone $(\mathrm{n}=1) ; 5)$ Ultrasound $(\mathrm{n}=2) ; 6)$ Vibratory stimulation $(\mathrm{n}=2)$; and 7) Virtual reality $(n=1)$.

\section{Complex interventions}


Sereda et al. Prioritising topics for systematic review

308 Twenty-four trials of complex interventions were identified including: 1) Heidelberg Neuro-

309 Music Therapy (n=2); 2) Perceptual/cognitive training (n=4); 3) Progressive Tinnitus

310 Management (PTM, n=4); 4) Tinnitus Retraining Therapy (TRT, including modified TRT;

$311 \mathrm{n}=9)$; 5) Combination of psychological approaches with other management strategies $(\mathrm{n}=3)$;

312 6) bimodal treatment involving TRT with EMDR and TRT with CBT (n=1); and 7) a

313 combination of sound based, educational and integrated medicine therapies $(n=1)$.

\section{Priority reviews on tinnitus}

315 Three high priority reviews were identified based on the pre-defined priority criteria. Those

316 were: 1) sound therapy using amplification devices and/or sound generators for tinnitus; 2)

317 betahistine; 3) CBT.

318 Sound therapy met the first three primary priority criteria, the existing Cochrane reviews

319 concluded a lack of evidence of clinical effectiveness (Hoare et al., 2014a, Hobson et al.,

320 2012) and new trials were identified. Our recommendation was that a priority Cochrane

321 review should include amplification only devices, combination devices (combined

322 amplification and sound generation), and sound generators. Suggested comparisons for

323 inclusion were: 1) Amplification only vs waiting-list control, placebo, education/information

324 only with no device; 2) Combination devices vs waiting-list control, placebo,

325 education/information only with no device, amplification only, sound generator only; 3 )

326 Sound generator only vs waiting-list control, placebo, education/information only with no

327 device. Trials that have conditions that explicitly included counselling (such as TRT, PTM,

328 Neuromonics) should be excluded. Counselling was defined according to Culley and Bond

329 (2011) as a process that aims to empower patients to reach decisions and take actions for

330 themselves. Establishing a therapeutic relationship, clarifying and defining problems,

331 planning actions, and managing expectations are all key features of the approach. Education 
Sereda et al. Prioritising topics for systematic review

332 and information giving can be entirely one-way, whereas counselling is about empowerment

333 and enabling patients to arrive at their own solutions using their own internal resources.

334 Therefore, unless there were explicit efforts and description of a process towards

335 empowerment in trial reports, and a trained therapist delivered it, then it was not considered

336 counselling.

337 Betahistine also met the first three primary priority criteria and there is no existing Cochrane

338 review. We identified six trials for consideration. Comparisons should include placebo, no

339 intervention, education and information only. However, it should be noted that only three

340 trials include the above comparisons $(\mathrm{n}=3)$ and the others would not be suitable for synthesis.

341 Subgroup analyses with and without Ménière's disease should also be considered, but we

342 note that there is an existing Cochrane review on Betahistine for Ménière's disease or

343 syndrome which has impact on tinnitus symptom severity as a secondary outcome (Van Esch

344 et al., 2018).

345 CBT met the first three primary priority criteria. Although there is an existing Cochrane

346 review (Martinez-Devesa et al., 2010) it is now outdated and does not include all cognitive,

347 and/or behavioural interventions (Acceptance and Commitment Therapy (ACT) and

348 Mindfulness-based therapies, described as different 'waves' of CBT). A Cochrane review

349 examining all cognitive and behavioural approaches for tinnitus is currently ongoing (Fuller

350 et al., 2017b).

351 Further priorities (meeting fewer priority criteria) included: 1) Gingko biloba; 2) anxiolytics;

352 3) hypnotics; 4) antiepileptics; 5) neuromodulation.

353 Gingko biloba met the first two primary priority criteria. The existing Cochrane review

354 concluded a lack of evidence for effectiveness (Hilton et al., 2013) and new trials were

355 identified. Suggested comparisons include placebo, no intervention, education and 
Sereda et al. Prioritising topics for systematic review

356

357

68

information only. Anxiolytics met the first two primary criteria and there is no existing

Cochrane review. Nine trials have been identified which may be eligible. Suggested

comparisons are placebo, no intervention, education and information only. Hypnotics meets

the first two primary criteria and there is no existing Cochrane review. Eight trials have been identified which may be eligible for inclusion. Suggested comparisons are placebo, no intervention, education and information only. Antiepileptics met the first two primary criteria and there is no existing Cochrane review. Eleven trials have been identified. Suggested comparisons include placebo, no intervention, education and information only.

Neuromodulation met two primary criteria including being in scope of the NICE guidelines. However, a Cochrane review of neuromodulation for tinnitus is currently ongoing (Hoare et al., 2015).

\section{7}

\section{CONCLUSIONS}

This technical report highlights a comprehensive exercise we undertook to prioritise topics of unmet need for high-quality systematic review in tinnitus management.

Importantly, these priority reviews will respond to unanswered questions identified in current and developing clinical practice guidelines for tinnitus. Three high priority reviews are recommended: 1) sound therapy using amplification devices and/or sound generators for tinnitus; 2) betahistine; 3) Cognitive Behaviour Therapy. Further priorities are: 4) Gingko biloba; 5) anxiolytics; 6) hypnotics; 7) antiepileptics; 8) neuromodulation.

Applying a prioritisation process ensures that resources are invested most effectively in work that meets the needs of funders and stakeholders and addresses known discrepancies or gaps in clinical knowledge. This particular prioritisation work focused on UK clinical practice for 
Sereda et al. Prioritising topics for systematic review

380

381

382

383

384

385

386

387

388

389

390

391

392

393

394

395

396

397

398

399

400

401

402

tinnitus and therefore the relevant priority criteria, such as availability of the intervention

within the NHS and inclusion in the scope of the NICE tinnitus guideline. However, the

process can easily be adapted to a range of international, national or local settings and

priorities. For example, regional or country-specific clinical practice can be taken into

consideration as well as guidelines at the national, regional or international level (e.g.

European or country-specific) when formulating the priority criteria.

The scoping exercise described here has already resulted in the expedited production of two Cochrane systematic reviews (Sereda et al., 2018; Wegner et al., 2018) in part to inform the NICE guideline on tinnitus which is currently under development. A further three priority reviews are currently in progress (Fuller et al., 2017b; Hoare et al. 2015; and Gingko biloba protocol in preparation).

\section{Acknowledgements}

MS, DB, IP, and DJH are funded by the National Institute for Health Research (NIHR)

Biomedical Research Centre programme. DAH is an NIHR Senior Investigator. The views expressed are those of the authors and not necessarily those of the NIHR, the NHS, or the

Department of Health and Social Care. RFFC is funded through The Netherlands

Organisation for Scientific Research (NWO); Innovational Research Incentives Scheme Veni.

We would like to thank Jenny Bellorini and Martin Burton (Cochrane ENT) for their comments on the review process and the report. We would also like to thank Sandra Smith and Snigdha Dutta for their assistance in the manuscript preparation. 
Sereda et al. Prioritising topics for systematic review

403

404

405

406

407

408

409

410

411

412

413

414

415

416

417

418

419

420

421

422

423

424

425

\section{References}

Ajayi, O. V., Phillips, J. S., Laopaiboon, M., McFerran, D. 2014. Melatonin for tinnitus. Cochrane Database of Systematic Reviews, 12: CD011435. https://doi.org/10.1002/14651858.CD011435

Arksey, H., O’Malley L. 2005. Scoping studies: towards a methodological framework. International Journal of Social Research Methodology: Theory \& Practice, 8: 19-32. https://doi.org/10.1080/1364557032000119616

Axelsson, A., Ringdahl, A. 1989. Tinnitus--a study of its prevalence and characteristics. British Journal of Audiology, 23(1): 53-62. https://doi.org/10.3109/03005368909077819

Baldo, P., Doree, C., Lazzarini, R., Molin, P., McFerran, D. 2012. Antidepressants for patients with tinnitus (Review). Cochrane Database of Systematic Reviews, 9: CD003853. https://doi.org/10.1002/14651858.CD003853.pub3

Bennett, M., Kertesz, T., Perleth, M., Yeung, P., Lehm, J., Lehm, J. P. 2012. Hyperbaric oxygen for idiopathic sudden sensorineural hearing loss and tinnitus (Review). Cochrane Database of Systematic Reviews, 10: CD004739. https://doi.org/10.1002/14651858.CD004739.pub4

British Society of Audiology. 2015. Tinnitus in Children Practice Guidance. Retrieved May 18, 2018, from http://www.thebsa.org.uk/now-available-new-bsa-tinnitus-in-childrenpractice-guidance/

Cima, R.F.F., Mazurek, B., Haider, H., Kikidis, D., Lapira, A., Noreña, A., Hoare, D.J. 2019. A multidisciplinary guideline for tinnitus: diagnostics, assessment, and treatment. $H N O$, 67 (Suppl 1): 10-42. https://doi.org/10.1007/s00106-019-0633-7 
Sereda et al. Prioritising topics for systematic review

426 Cochrane ENT group. 2019. Scoping projects. Retrieved July 24, 2019, from

427 https://ent.cochrane.org/our-evidence/prioritisation/scoping-projects.

428 Culley, S., Bond, T. 2011. Integrative counselling skills in action. Sage Publications Ltd; 3rd $429 \quad$ edition.

430 Davis, A., \& El Refaie, A. 2000. Epidemiology of tinnitus. In R. Tyler (Ed.), Tinnitus

431 Handbook. San Diego: Singular Publishing Group.

432 Department of Health. 2009. Provision of Services for Adults with Tinnitus: A Good Practice $433 \quad$ Guide.

434 https://doi.org/http://webarchive.nationalarchives.gov.uk/20130107105354/http:/www.d 435 h.gov.uk/prod_consum_dh/groups/dh_digitalassets/documents/digitalasset/dh_093810.p $436 \quad \underline{\mathrm{df}}$

437 El-Shunnar, S. K., Hoare, D. J., Smith, S., Gander, P. E., Kang, S., Fackrell, K., and Hall, D. 438 A. 2011. Primary care for tinnitus: Practice and opinion among GPs in England. Journal 439 of Evaluation in Clinical Practice, 17(4): 684-692. https://doi.org/10.1111/j.1365$440 \quad \underline{2753.2011 .01696 . x}$

441 Fuller, T.E., Haider, H.F., Kikidis, D., Lapira, A., Mazurek, B., Norena, A., Rabau, S., 442 Lardinois, R., Cederroth, C.R., Edvall, N.K., Brueggemann, P.G., Rosing, S.N., 443 Kapandais, A., Lungaard, D., Hoare, D.J., Cima, R.F. 2017a. Different Teams, Same 444 Conclusions? A Systematic Review of Existing Clinical Guidelines for the Assessment 445 and Treatment of Tinnitus in Adults. Frontiers in Psychology, 8:206. https://doi: 10.3389/fpsyg.2017.00206. eCollection 2017.

447 Fuller, T.E., Cima, R., Langguth, B., Mazurek, B., Waddell, A., Hoare, D. J., and Vlaeyen, J. 448 W. S. 2017b. Cognitive behavioural therapy for tinnitus. Cochrane Database of $449 \quad$ Systematic Reviews, 4: CD012614. https://doi.org/10.1002/14651858.CD012614 
Sereda et al. Prioritising topics for systematic review

450 Greenwell, K., Sereda, M., Coulson, N, El Refaie, A., Hoare, D.J. 2016. A systematic review

of techniques and effects of self-help interventions for tinnitus: Application of

$\underline{\text { taxonomies from health psychology. Int J Audiol, Suppl 3: S79-89. }}$

https://doi.org/10.3109/14992027.2015.1137363

Hall, D.A., Láinez, M.J., Newman, C.W., Sanchez, T.G., Egler, M., Tennigkeit, F., Koch, M., Langguth, B. 2011. Treatment options for subjective tinnitus: self reports from a sample of general practitioners and ENT physicians within Europe and the USA. BMC Health Services Research, 11: 302. https://doi.org/10.1186/1472-6963-11-302

Higgins, J.P.T. and Green, S. 2011. Cochrane Handbook for Systematic Reviews of Interventions Version 5.1.0 [updated March 2011]. The Cochrane Collaboration. Retrieved May 18, 2018, from www.cochrane-handbook.org

Hilton, M., Zimmermann, E.F, Stuart, E. 2013. Ginkgo biloba for tinnitus. Cochrane Database of Systematic Reviews, 3: CD003852.

Hoare, D. J., Edmondson-Jones, M., Sereda, M., Akeroyd, M. A., and Hall, D. 2014. Amplification with hearing aids for patients with tinnitus and co-existing hearing loss

Hoare, D. J., Whitham, D., Henry, J. A., and Shorter, G. W. 2015. Neuromodulation (desynchronisation) for tinnitus in adults. Cochrane Database of Systematic Reviews, 6: (Review). Cochrane Database of Systematic Reviews, 1: CD010151. https://doi.org/10.1002/14651858.CD010151 
475

Sereda et al. Prioritising topics for systematic review

474 Hoekstra, C. E. L., Rynja, S. P., Van Zanten, G. A., and Rovers, M. 2011. Anticonvulsants

Li, Y., Zeng, R. F., and Zheng, D. 2016. Acupuncture for tinnitus. Cochrane Database of Systematic Reviews, 11: CD008149. https://doi.org/10.1002/14651858.CD008149.pub2

Maldonado Fernández, M., Shin, J., Scherer, R. W., and Murdin, L. 2015. Interventions for tinnitus in adults: an overview of systematic reviews. Cochrane Database of Systematic Reviews, 1: CD011795. https://doi.org/10.1002/14651858.CD011795

Martinez-Devesa, P., Perera, R., Theodoulou, M., and Waddell, A. 2010. Cognitive behavioural therapy for tinnitus. Cochrane Database of Systematic Reviews, 9: CD005233. https://doi.org/10.1002/14651858.CD005233.pub3 
Sereda et al. Prioritising topics for systematic review

497

498

499

500

501

502

503

504

505

506

507

508

509

510

511

512

513

514

47

48

49

50

51

52

53 519

review of the reporting of tinnitus prevalence and severity. Hearing Research, 337: 70-

\section{9. https://doi.org/10.1016/j.heares.2016.05.009}

McFerran, D., Hoare, D.J., Carr, S., Ray, J., Stockdale, D. 2018. Tinnitus services in the United Kingdom: a survey of patient experiences. BMC Health Services Research, 18(1): 110. https://doi.org/110.1186/s12913-018-2914-3.

Meikle, M., Taylor-Walsh, E. 1984. Characteristics of tinnitus and related observations in over 1800 tinnitus clinic patients. The Journal of Laryngology \& Otology, 9: 17-21. https://doi.org/10.1017/S1755146300090053

Meng, Z., Liu, S., Zheng, Y., and Phillips, J. S. 2011. Repetitive transcranial magnetic stimulation for tinnitus. Cochrane Database of Systematic Reviews, 10: CD007946. https://doi.org/10.1002/14651858.CD007946.pub2

Methley, A.M., Campbell, S., Chew-Graham, C., McNally, R., Cheraghi-Sohi, S. 2014. PICO, PICOS and SPIDER: a comparison study of specificity and sensitivity in three search tools for qualitative systematic reviews. BMC Health Services Research, 14:579.

https://doi.org/10.1186/s12913-014-0579-0

Morata, T.C., Hickson, L., Wong, L. 2017. The IJA system for systematic reviews: "the whys and hows". Int J Audiol, 56(4): 213-214. https://doi.org/10.1080/14992027.2016.1275044

515 Peng, Z., Chen, X., Gong, S., and Chen, C. 2012. Low-level laser therapy for tinnitus.

$516 \quad$ Cochrane Database of Systematic Reviews, 9: CD009811 https://doi.org/10.1002/14651858.CD009811

518 Person, O. C., Puga, M. E. S., and da Silva, E. M. K. 2016. Zinc supplementation for tinnitus (Review). Cochrane Database of Systematic Reviews, 11: CD009832. 
Sereda et al. Prioritising topics for systematic review

520

521

522

523

524

525

526

527

528

529

530

531

532

533

534

535

536

537

538

539

540

541

542

543

https://doi.org/10.1002/14651858.CD009832

Phillips, J. S., and McFerran, D. 2010a. Tinnitus retraining therapy (TRT) for tinnitus patients. Cochrane Database of Systematic Reviews, 3: CD007330. https://doi.org/10.1002/14651858.CD007330

Phillips, J. S., and McFerran, D. 2010b. Neurophysiological model-based treatments for tinnitus. Cochrane Database of Systematic Reviews, 1: CD008248. https:// $\underline{10.1002 / 14651858 . C D 008248 . p u b 2}$

Schünemann, H., Brożek, J., Guyatt, G., and Oxman A (editors). 2013. The GRADE Working Group. GRADE Handbook for Grading Quality of Evidence and Strength of Recommendations [Updated October 2013]. Retrieved May 18, 2018, from http:/gdt.guidelinedevelopment.org/app/handbook/handbook.html

Sereda, M., Xia, J., El Refaie, A., Hall, D.A., Hoare, D.J. 2018. Sound therapy (using amplification devices and/or sound generators) for tinnitus. Cochrane Database of Systematic Reviews, 12: CD013094. https://doi.org/10.1002/14651858.CD013094.pub2

Stockdale, D., McFerran, D., Brazier, P., Pritchard, C., Kay, T., Dowrick, C., Hoare, D. J. 2017. An economic evaluation of the healthcare cost of tinnitus management in the UK. BMC Health Services Research, 17(1): 1-9. https://doi.org/10.1186/s12913-017-2527-2

Tricco, A.C., Lillie, E., Zarin, W., O'Brien, K.K., Colquhoun, H., Levac, D., Moher, D., Peters, M.D.J., Horsley, T., Weeks, L., Hempel, S., Ak1, E.A., Chang, C., McGowan, J., Stewart, L., Hartling, L., Aldcroft, A., Wilson, M.G., Garritty, C., Lewin, S., Godfrey, C.M., Macdonald, M.T., Langlois, E.V., Soares-Weiser, K., Moriarty, J., Clifford, T., Tunçalp, Ö., Straus, S.E.. 2018. PRISMA extension for scoping reviews (PRISMAScR): checklist and explanation. Annals of Internal Medicine, 169(7):467-473. 
Sereda et al. Prioritising topics for systematic review

544 van Esch, B., van der Zaag-Loonen, H. J., Bruintjes, T., Murdin, L., James, A., van Benthem, 545 P. P. 2018. Betahistine for Ménière’s disease or syndrome. Cochrane Database of $546 \quad$ Systematic Reviews, 1: CD012914. https://doi: 10.1002/14651858.CD012914

547 Wegner, I., Hall, D.A., Smit, A.L., McFerran, D., Stegeman, I. 2018. Betahistine for tinnitus. $548 \quad$ Cochrane Database of Systematic Reviews, 12: CD013093.

$549 \quad$ https://doi.org/14651858.CD013093.pub2

550

551

552

553

554 FIGURE LEGEND

555 Figure 1. Flow diagram illustrating search strategy and scoping review stages

556

557 SUPPLEMENTAL MATERIAL

558 Supplemental material 1. Summary of priority criteria for each of the interventions 559 
1 MESH DESCRIPTOR Tinnitus EXPLODE ALL AND

INREGISTER

2 tinnit* AND INREGISTER

3 \#1 OR \#2 AND INREGISTER

Cochrane ENT Trials Register (via the Cochrane

Register of Studies)

Records returned: $n=1080$

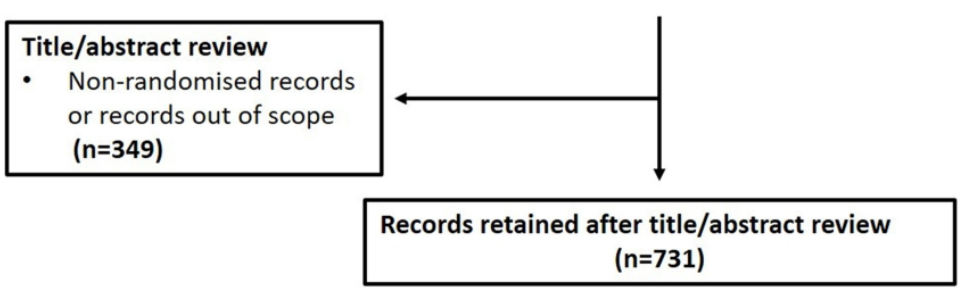

Data extraction

- Duplicates ( $n=127)$

- Records out of scope ( $n=11)$

- Non-randomised studies $(n=86)$

- Conference abstracts with no published results $(n=70)$

- Abstract/full text requires translation $(\mathbf{n}=\mathbf{1 5})$

- Abstract/full text not available $(n=9)$

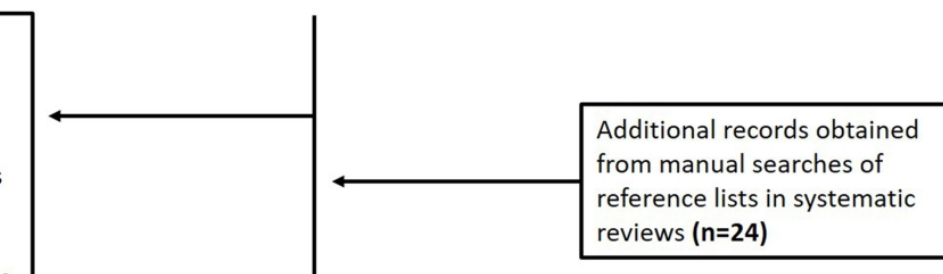

New (not covered in existing Cochrane reviews) RCTs with published results (PICOS extracted)

$(n=365)$

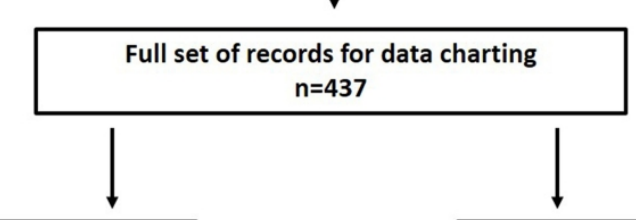

Trial registrations (no results available; trial identifier, date of registration, trial status, main intervention and comparisons extracted) $(n=51)$

Figure 1. Flow diagram illustrating search strategy and scoping review stages $177 \times 205 \mathrm{~mm}(600 \times 600 \mathrm{DPI})$ 
Supplemental material 1: Summary of priority criteria for each of the interventions

Summary of interventions with ratings according to the primary and secondary criteria for prioritisation. To aid prioritisation decisions, four primary criteria were considered: 1 . Whether the intervention is available for tinnitus management within the NHS; 2 . Whether the intervention is within the scope of the NICE tinnitus guidelines that are currently in development; 3 . Whether there was 'no recommendation' or disagreement in recommendations across current management guidelines; and 4. Whether existing Cochrane systematic reviews concluded there was a lack of evidence, but new RCTs are now available or there is no Cochrane review.

In addition, four secondary criteria considered: 5 . Whether the intervention has been prioritised in the James Lind Alliance Priority Setting Partnership for tinnitus as a 'top 10' uncertainty; 6 . The number of new RCTS identified; 7. Whether interventions are referred to in the TINNET European clinical practice guideline; and 8 . Whether there is evidence for variability in clinical practice, within or across countries.

\begin{tabular}{|c|c|c|c|c|c|c|c|c|}
\hline \multirow[b]{2}{*}{ Intervention } & \multicolumn{4}{|c|}{ Primary criteria } & \multicolumn{4}{|c|}{ Secondary criteria } \\
\hline & $\begin{array}{c}1 . \\
\mathrm{NHS}\end{array}$ & $\begin{array}{l}2 . \\
\text { NICE }\end{array}$ & $\begin{array}{c}3 . \\
\text { Guidelines }\end{array}$ & $\begin{array}{c}4 . \\
\text { Cochrane } \\
\text { needed }\end{array}$ & $\begin{array}{c}5 . \\
\text { JLA }\end{array}$ & $\begin{array}{c}6 . \\
\text { New } \\
\text { RCTs }\end{array}$ & $\begin{array}{c}7 . \\
\text { TINNET }\end{array}$ & $\begin{array}{c}8 . \\
\text { Variability }\end{array}$ \\
\hline \multicolumn{9}{|c|}{ Pharmacological approaches - Alimentary tract and metabolism } \\
\hline Drugs for functional gastrointestinal disorders & NO & NO & YES & YES & YES & 4 & NO & YES \\
\hline Antiemetics and antinauseants & YES & NO & YES & YES & YES & 1 & NO & YES \\
\hline Vitamins - Ascorbic acid (Vitamin C) & NO & NO & YES & YES & YES & 1 & YES & YES \\
\hline Vitamins - other plain Vitamin preparations & NO & NO & YES & YES & YES & 2 & YES & YES \\
\hline $\begin{array}{l}\text { Vitamins - Vitamin B-complex, including } \\
\text { combinations }\end{array}$ & NO & NO & YES & YES & YES & 2 & YES & YES \\
\hline Mineral supplements - Zinc & NO & NO & YES & NO & YES & 0 & YES & YES \\
\hline Mineral supplements - Magnesium & NO & NO & YES & YES & YES & 1 & YES & YES \\
\hline
\end{tabular}




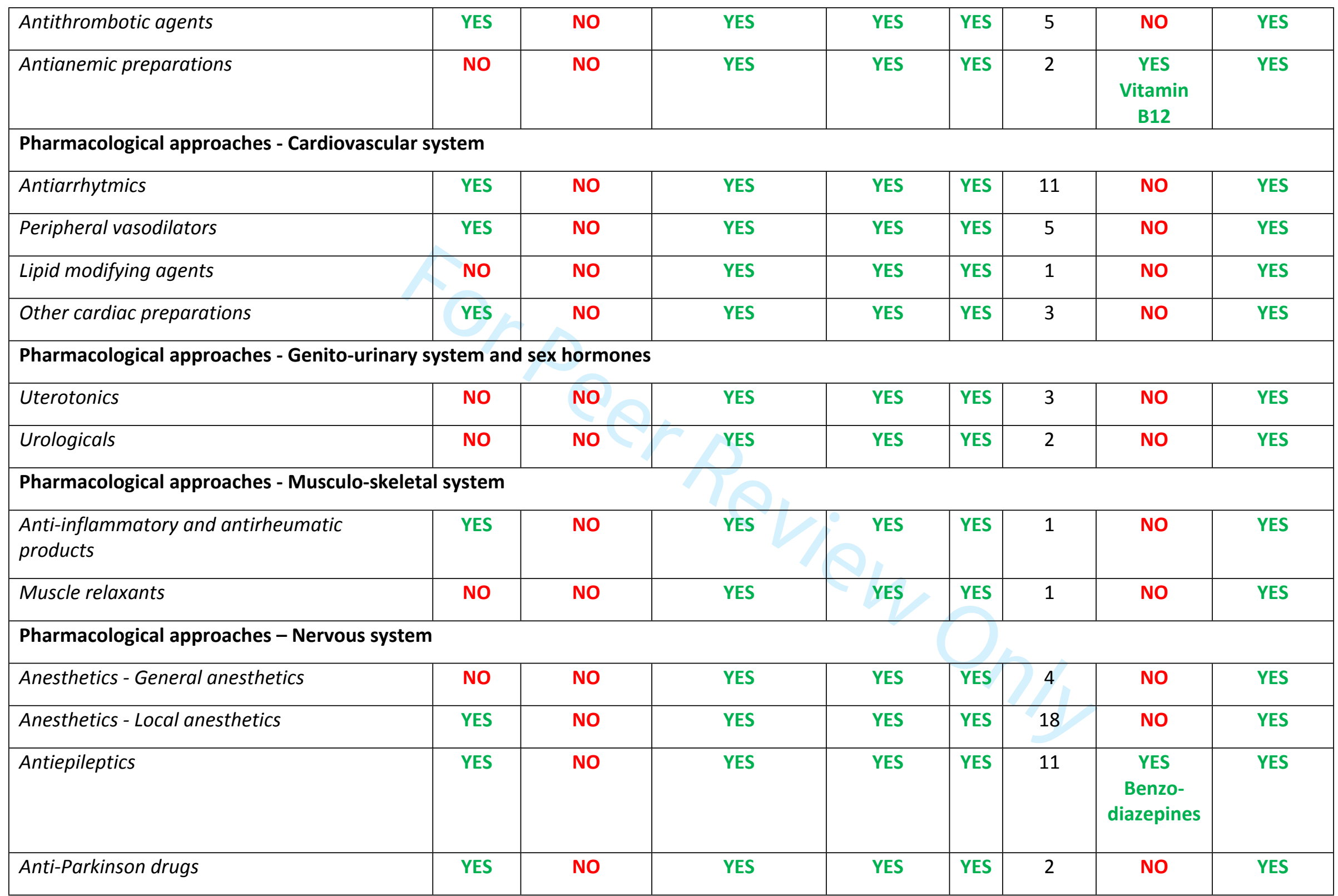




\begin{tabular}{|c|c|c|c|c|c|c|c|c|}
\hline Psycholeptics - Antipsychotics & YES & NO & YES & YES & YES & 2 & NO & YES \\
\hline Psycholeptics - Anxiolytics & YES & NO & YES & YES & YES & 8 & NO & YES \\
\hline Hypnotics and sedatives & YES & NO & YES & $\begin{array}{c}\text { YES } \\
\text { Melatonin }\end{array}$ & YES & 8 & $\begin{array}{c}\text { YES } \\
\text { Melatonin }\end{array}$ & YES \\
\hline Psychoanaleptics - Antidepressants & YES & NO & YES & YES & YES & 4 & YES & YES \\
\hline Psychostimulants and nootropics & YES & NO & YES & YES & YES & 1 & NO & YES \\
\hline Anti-dementia drugs & YES & $\begin{array}{c}\text { NO } \\
\text { Ginkgo } \\
\text { biloba }\end{array}$ & YES & YES & YES & 6 & YES & YES \\
\hline $\begin{array}{l}\text { Other nervous system drugs - Drugs used in } \\
\text { addictive disorders }\end{array}$ & NO & NO & YES & YES & YES & 3 & NO & YES \\
\hline Antivertigo preparations & YES & $\begin{array}{c}\text { YES } \\
\text { Betahistine }\end{array}$ & ES & YES & YES & 11 & NO & YES \\
\hline Combinations of medications & NO & NO & YES & YES & YES & 1 & NO & YES \\
\hline \multicolumn{9}{|c|}{ Pharmacological approaches - respiratory system } \\
\hline Respiratory stimulants & YES & NO & YES & YES & YES & 1 & NO & YES \\
\hline \multicolumn{9}{|c|}{ Pharmacological approaches - Systemic hormonal preparations, excluding sex hormones and insulins } \\
\hline $\begin{array}{l}\text { Pituitary and hypothalamic hormones and } \\
\text { analogues }\end{array}$ & NO & NO & YES & YES & YES & 1 & NO & YES \\
\hline Corticosteroids for systemic use & YES & NO & YES & YES & YES & 10 & NO & YES \\
\hline \multicolumn{9}{|l|}{ Pharmacological approaches - various } \\
\hline Medical gases - Oxygen & YES & NO & YES & YES & YES & 2 & NO & YES \\
\hline
\end{tabular}




\begin{tabular}{|c|c|c|c|c|c|c|c|c|}
\hline \multicolumn{9}{|c|}{ Pharmacological approaches - non-classified medications (i.e. experimental) } \\
\hline Amino-oxyacetic acid & NO & NO & YES & YES & YES & 2 & NO & YES \\
\hline Glutamate & NO & NO & YES & YES & YES & 1 & NO & YES \\
\hline Neramexane & NO & NO & YES & YES & YES & 6 & NO & YES \\
\hline Nerve growth factor & NO & NO & YES & YES & YES & 2 & NO & YES \\
\hline Dextran 40 & NO & NO & YES & YES & YES & 1 & NO & YES \\
\hline Selurampanel & NO & NO & YES & YES & YES & 1 & NO & YES \\
\hline Vestipitant & NO & NO & YES & YES & YES & 1 & NO & YES \\
\hline \multicolumn{9}{|l|}{ Sound-based interventions } \\
\hline Acoustic CR Neuromodulation & NO & YES & YES & YES & $?$ & 3 & YES & YES \\
\hline Amplification only devices & YES & YES & YES & YES & YES & 8 & YES & YES \\
\hline $\begin{array}{l}\text { Combination devices (i.e. combined } \\
\text { amplification and sound generation) }\end{array}$ & YES & YES & YES & YES & YES & 5 & YES & YES \\
\hline Phase-tailored sound treatment & NO & NO & YES & YES & NO & 1 & NO & YES \\
\hline $\begin{array}{l}\text { Sound generators only devices (sometimes } \\
\text { referred to as 'maskers') }\end{array}$ & YES & YES & YES & YES & NO & 20 & YES & YES \\
\hline Spectrally tailored sound treatment & NO & NO & YES & YES & NO & 3 & YES & YES \\
\hline Auditory training & NO & YES & YES & YES & NO & 4 & NO & YES \\
\hline \multicolumn{9}{|l|}{ Psychology-based interventions } \\
\hline Cognitive/Behavioural approaches & YES & YES & NO & YES & YES & 36 & YES & YES \\
\hline Counselling & YES & YES & NO & YES & NO & 3 & YES & YES \\
\hline
\end{tabular}




\begin{tabular}{|c|c|c|c|c|c|c|c|c|}
\hline \multicolumn{9}{|l|}{ Complex interventions } \\
\hline Heidleberg Neuro-Music Therapy & NO & NO & YES & YES & NO & 2 & NO & YES \\
\hline Perceptual/Cognitive training & NO & NO & YES & YES & NO & 4 & NO & YES \\
\hline Progressive Tinnitus Management & NO & YES & YES & YES & NO & 4 & NO & YES \\
\hline Tinnitus Retraining Therapy & NO & YES & YES & YES & NO & 9 & YES & YES \\
\hline Various - CBT plus biofeedback & NO & NO & YES & YES & NO & 2 & NO & YES \\
\hline Various - CBT plus TRT (Cima) & NO & NO & YES & YES & NO & 1 & NO & YES \\
\hline \multicolumn{9}{|l|}{ Magnetic stimulation } \\
\hline Transcranial Magnetic Stimulation & NO & NO & YES & YES & NO & 39 & YES & YES \\
\hline $\begin{array}{l}\text { Various - electromagnetic stimulation of the } \\
\text { ear }\end{array}$ & NO & NO & YES & YES & NO & 1 & No & YES \\
\hline Various - ear magnets & NO & NO & YES & YES & NO & 1 & NO & YES \\
\hline \multicolumn{9}{|l|}{ Electrical stimulation } \\
\hline Cochlear implants & NO & NO & YES & YES & NO & 3 & YES & YES \\
\hline $\begin{array}{l}\text { Transcranial Alternating Current Stimulation } \\
\text { (tACS) }\end{array}$ & NO & NO & YES & YES & NO & 1 & YES & YES \\
\hline Transcranial Direct Current Stimulation & NO & NO & YES & YES & NO & 11 & YES & YES \\
\hline Transcutaneous electrical stimulation & NO & NO & YES & YES & NO & 2 & NO & YES \\
\hline Vagus nerve stimulation & NO & NO & YES & YES & NO & 2 & YES & YES \\
\hline $\begin{array}{l}\text { Various - electrical stimulation of the ear } \\
\text { (tympanic membrane) }\end{array}$ & NO & NO & YES & YES & NO & 1 & NO & YES \\
\hline
\end{tabular}




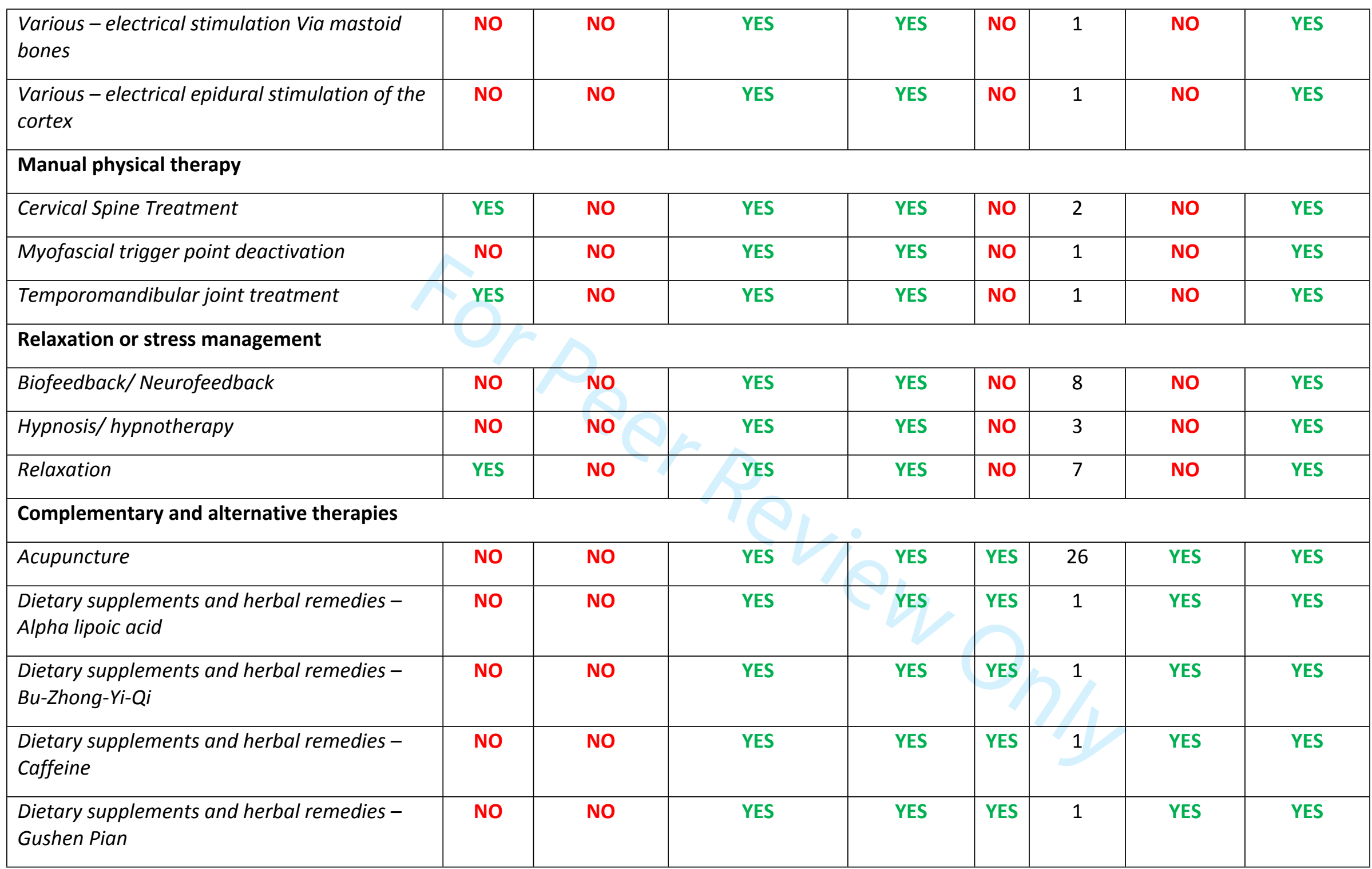




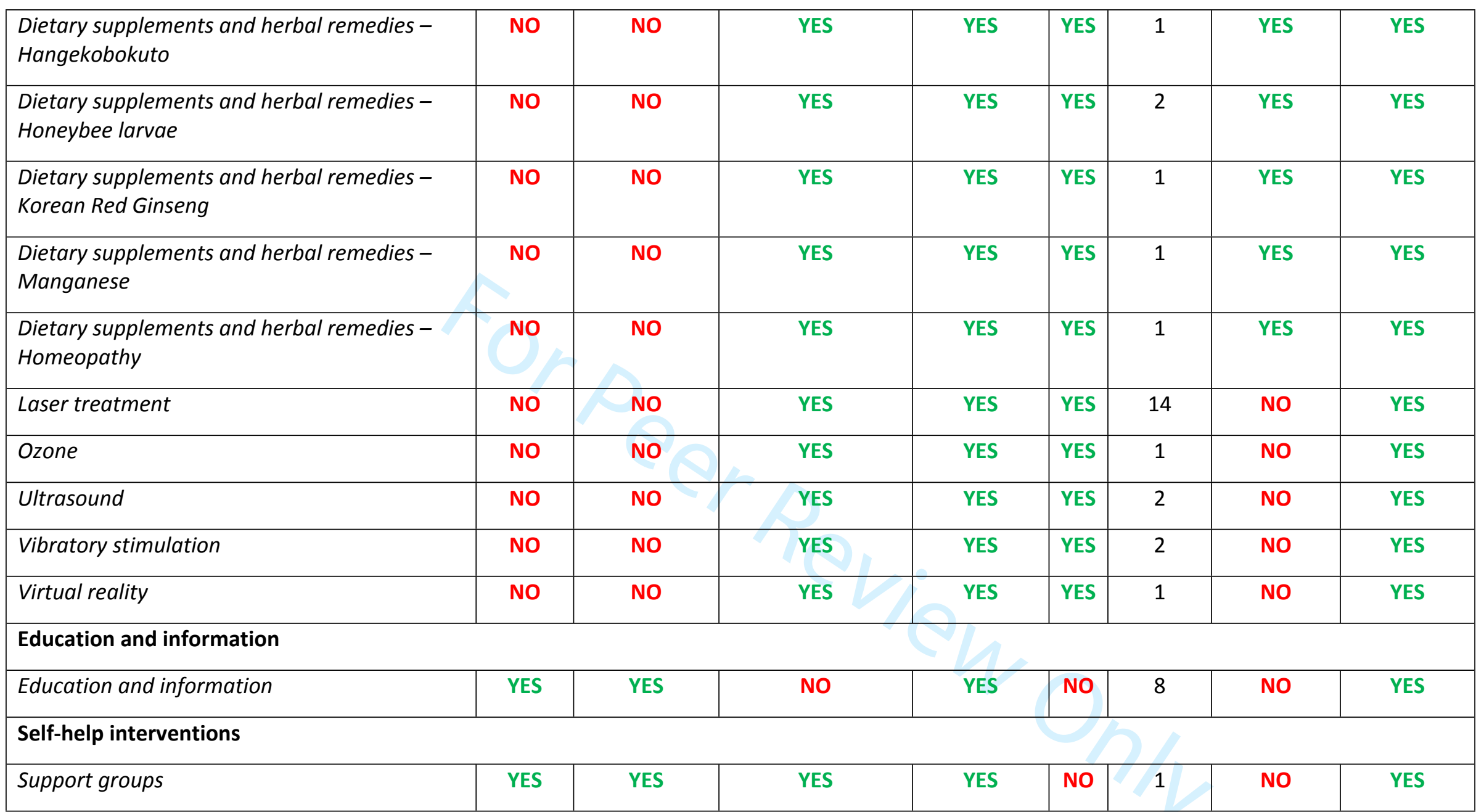

